TRANSACTIONS OF THE

AMERICAN MATHEMATICAL SOCIETY

Volume 348, Number 11, November 1996

\title{
THE MORSE SPECTRUM OF LINEAR FLOWS ON VECTOR BUNDLES
}

\author{
FRITZ COLONIUS AND WOLFGANG KLIEMANN
}

Dedicated to J. L. Massera

\begin{abstract}
For a linear flow $\Phi$ on a vector bundle $\pi: E \rightarrow S$ a spectrum can be defined in the following way: For a chain recurrent component $\mathcal{M}$ on the projective bundle $\mathbb{P} E$ consider the exponential growth rates associated with (finite time) $(\varepsilon, T)$-chains in $\mathcal{M}$, and define the Morse spectrum $\Sigma_{M o}(\mathcal{M}, \Phi)$ over $\mathcal{M}$ as the limits of these growth rates as $\varepsilon \rightarrow 0$ and $T \rightarrow \infty$. The Morse spectrum $\Sigma_{M o}(\Phi)$ of $\Phi$ is then the union over all components $\mathcal{M} \subset \mathbb{P} E$. This spectrum is a synthesis of the topological approach of Selgrade and Salamon/Zehnder with the spectral concepts based on exponential growth rates, such as the Oseledec spectrum or the dichotomy spectrum of Sacker/Sell. It turns out that $\Sigma_{M o}(\Phi)$ contains all Lyapunov exponents of $\Phi$ for arbitrary initial values, and the $\Sigma_{M o}(\mathcal{M}, \Phi)$ are closed intervals, whose boundary points are actually Lyapunov exponents. Using the fact that $\Phi$ is cohomologous to a subflow of a smooth linear flow on a trivial bundle, one can prove integral representations of all Morse and all Lyapunov exponents via smooth ergodic theory. A comparison with other spectral concepts shows that, in general, the Morse spectrum is contained in the topological spectrum and the dichotomy spectrum, but the spectral sets agree if the induced flow on the base space is chain recurrent. However, even in this case, the associated subbundle decompositions of $E$ may be finer for the Morse spectrum than for the dynamical spectrum. If one can show that the (closure of the) Floquet spectrum (i.e. the Lyapunov spectrum based on periodic trajectories in $\mathbb{P} E$ ) agrees with the Morse spectrum, then one obtains equality for the Floquet, the entire Oseledeč, the Lyapunov, and the Morse spectrum. We present an example (flows induced by $C^{\infty}$ vector fields with hyperbolic chain recurrent components on the projective bundle) where this fact can be shown using a version of Bowen's Shadowing Lemma.
\end{abstract}

\section{INTRODUCTION}

For a linear, time invariant differential equation $\dot{x}=A x$ in $\mathbb{R}^{d}$ the corresponding eigenspace structure can be described in two different ways: Let $V$ be the sum of (generalized) eigenspaces of $A$ corresponding to all eigenvalues $\lambda_{i}$ with equal real

Received by the editors January 25, 1994 and, in revised form, March 31, 1995.

1991 Mathematics Subject Classification. Primary 58F25, 34C35, 34D08.

Key words and phrases. Chain recurrence, ergodic theory, Lyapunov exponents, dichotomy spectrum, topological spectrum, Oseledeč spectrum, Floquet spectrum, hyperbolic flows, shadowing lemma.

This research was performed during a stimulating visit at the Institute for Mathematics and Its Applications, Minneapolis. It was partially supported by DFG under grant no. Co 124/8-2 and by ONR grant no. N00014-93-1-0868. 
part. Then

$$
x \in V \text { iff } \lambda^{+}(x)=\lambda^{-}(x)=\operatorname{Re} \lambda_{i},
$$

where $\lambda^{+}$and $\lambda^{-}$are the Lyapunov exponents of the solution $\Phi_{t} x$, defined by

$$
\lambda^{+}(x)=\limsup _{t \rightarrow \infty} \frac{1}{t} \log \left|\Phi_{t} x\right|, \lambda^{-}(x)=\limsup _{t \rightarrow-\infty} \frac{1}{t} \log \left|\Phi_{t} x\right| .
$$

Besides this characterization through exponential growth rates, the subspace $V$ can be described topologically, by looking at the induced differential equation on the real projective space $\mathbb{P}^{d-1}$ : The projection of $V$ onto $\mathbb{P}^{d-1}$ is a component of the chain recurrent set of the projected flow.

For linear, time variant differential equations such a decomposition of $\mathbb{R}^{d}$ is, in general, not possible - this is the problem of Lyapunov regularity. Therefore, one has to use more general spectral concepts, which can be developed in various, convincing ways if the linear system is a part of a dynamical system, i.e. a cocycle over a base flow: One considers a linear flow $\Psi: \mathbb{R} \times E \rightarrow E$ on a vector bundle $\pi: E \rightarrow S$ and defines the Lyapunov exponents $\lambda(e)$ for $e \in E$ as above using the cocycle. The characterization via exponential growth rates is essentially used in the theory of exponential dichotomies (see e.g. [DK], [Cp], [SS2], [Sl]) and in Oseledeč theory [Os] in order to obtain 'regular' spectral concepts for linear flows. In particular, the corresponding 'eigenspace structure', i.e. (continuous or measurable) subbundle decompositions of $E$, are obtained by looking at points characterized by their exponential growth behavior.

The topological approach has been used by Selgrade [Sg] and by Salamon and Zehnder [SZ]. For linear flows on vector bundles the exponential growth rates of trajectories in different chain recurrent components of the projected flow on the projective bundle $\mathbb{P} E$ may be equal (cf. e.g. the simple, but elucidating example in $[\mathrm{SZ}]$, p. 626, or the example in the context of bilinear control theory in [CK1], Example 4.11). Therefore, this approach yields a finer analysis of the 'eigenspace structure', i.e. here the chain recurrent components on $\mathbb{P} E$. Note however, that for a chain recurrent base space $S$ the set of spectral values obtained via the dichotomy spectrum of Sacker and Sell, which is based on exponential dichotomies, agrees with the set obtained from the topological characterization on the projective bundle.

The Morse spectrum, developed in this paper, is based on the topological approach. Over a chain recurrent component $\mathcal{M}$ of the projected flow on $\mathbb{P} E$ we consider the exponential growth rates associated with (finite time) $(\varepsilon, T)$-chains in $\mathcal{M}$ and define the Morse spectrum $\Sigma_{M o}(\mathcal{M}, \Psi)$ of the linear flow $\Psi$ over $\mathcal{M}$ as the limits of these growth rates as $\varepsilon \rightarrow 0$ and $T \rightarrow \infty$. The Morse spectrum $\Sigma_{M o}(\Psi)$ is then the union over all components $\mathcal{M} \subset \mathbb{P} E$. Thus this paper goes one step further in the program initiated by Conley [Cn], Selgrade [Sg] and Salamon and Zehnder [SZ]: It starts from the topological characterization, but defines a spectrum via exponential growth rates. Thus it can be viewed as a synthesis of both approaches. It turns out that the Morse spectrum contains the Lyapunov spectrum of the flow $\Psi$, and it is in general finer than the topological and the dynamical spectrum. Over a chain recurrent base space $S$ the sets of spectral values agree for all three concepts, but the associated subbundle decomposition (the 'eigenspace structure') of the Morse spectrum can be finer. An advantage of the Morse spectrum is its closeness to the Lyapunov spectrum: If it is possible to 'close' the defining $(\varepsilon, T)$-chains approximately via trajectories of the projected flow, then one may be able to show 
that the Lyapunov and the Morse spectrum agree. An example for this fact is presented in Section 6, using a refined version of Bowen's Shadowing Lemma, see also [CK6] for results on the Lyapunov spectrum of $L^{\infty}$-families of time varying matrices.

Another connection between the topological approach and the approach based on exponential growth rates has been established by Bronstein and Chernii [BC]. They showed that a decomposition into exponentially separated subbundles is equivalent to an attractor-repeller decomposition in the projective bundle. We will use their result in order to show that the intervals of the Morse spectrum over a chain recurrent base space are strictly ordered.

The contents of this paper are as follows: After the introductory Section 2, collecting some basic notions and facts on linear flows on vectorbundles, the central Section 3 introduces the concept of the Morse spectrum. It is shown that the periodic Morse spectrum (based on periodic $(\varepsilon, T)$-chains) consists of closed intervals, and it contains all Lyapunov exponents for arbitrary initial points. Furthermore, it enjoys an upper semicontinuity property with respect to perturbations of the flow.

In Section 4 we show that the boundary points of the Morse spectral intervals are attained as actual Lyapunov exponents for almost all initial values in the support of ergodic invariant measures on the projective bundle. This implies in particular that the periodic Morse spectrum and the Morse spectrum agree. The main tools for these results are the facts that every linear flow on a vector bundle is cohomologous to a subflow of a smooth linear flow on a trivial bundle, and that for smooth flows on these bundles the Krylov-Bogolyubov construction of invariant measures can be generalized to chains. Then the Morse spectrum can be analyzed using smooth ergodic theory, which also yields integral representations for all Lyapunov exponents.

Section 5 discusses the relations of the Morse spectrum to other spectral concepts, namely the topological spectrum [SZ], the dichotomy (or dynamical) spectrum [SS2], the Oseledec spectrum [Os], and the Lyapunov spectrum of the flow. One obtains the following chain of inclusions

$$
\begin{gathered}
\partial \Sigma_{d i c} \subset \partial \Sigma_{M o} \subset \bigcup_{\mu \text { ergodic }} \Sigma_{O s}(\mu) \subset \bigcup_{\mu \text { stationary }} \Sigma_{O s}(\mu) \\
\subset \Sigma_{L y} \subset \Sigma_{M o} \subset \Sigma_{t o p} \subset \Sigma_{d i c} .
\end{gathered}
$$

Over a chain recurrent base space the last two inclusions are equalities. Furthermore, the relations between the associated subbundle decompositions are analyzed.

It holds that (with $\Sigma_{F l}$ denoting the Floquet (i.e. periodic) spectrum of a flow)

$$
\Sigma_{F \ell} \subset \bigcup_{\mu \text { ergodic }} \Sigma_{O s}(\mu) \subset \Sigma_{L y} \subset \Sigma_{M o}
$$

Therefore, if one can show that $c \ell \Sigma_{F \ell}=c \ell \Sigma_{M o}$, then all four sets of spectral values agree. This property holds, if chains can be shadowed in such a way by actual trajectories on the projective bundle, that the chain exponent and the Lyapunov exponent are close. This idea is carried out in Section 6 for $C^{\infty}$ vector fields with hyperbolic chain recurrent components of the induced flow on the projective bundle. The main tool is a refined version of the continuous time Shadowing Lemma. 


\section{Basic Concepts for Flows on Vector Bundles}

A continuous time flow on a metric space $(S, d)$ is given by a continuous map

$$
\begin{aligned}
& \Psi: \mathbb{R} \times S \rightarrow S \quad \text { with } \\
& \Psi(0, p)=p, \Psi(t, \Psi(s, p))=\Psi(t+s, p)
\end{aligned}
$$

for all $t, s \in \mathbb{R}, p \in S$. As usual, we write $\Psi_{t}:=\Psi(t, \cdot)$. Note that each $\Psi_{t}$ is a homeomorphism in this setup. For $A \subset S$, the $\omega$-limit set of $A$ is given by

$$
\omega(A):=\left\{q \in S \text {; there are } p_{k} \in A, t_{k} \rightarrow \infty \text { with } \Psi\left(t_{k}, p_{k}\right) \rightarrow q \text { as } k \rightarrow \infty\right\},
$$

and similarly for $\omega^{*}(A)$ via $t_{k} \rightarrow-\infty$.

An $(\varepsilon, T)$-chain $\zeta$ of $\Psi$ is given by (cf. [Cn], Section II or $[\mathrm{Ak}]$ )

$$
\begin{aligned}
& n \in \mathbb{N}, T_{0}, \ldots, T_{n-1} \geq T, \text { and } p_{0}, \ldots, p_{n-1} \in S \text { such that } \\
& d\left(\Psi\left(T_{i}, p_{i}\right), p_{i+1}\right)<\varepsilon \text { for } i=0, \ldots, n-1 .
\end{aligned}
$$

An $(\varepsilon, T)$-chain is called periodic, if $p_{n}=p_{0}$. The chain recurrent set $\mathcal{R}(\Psi)$ is defined as $\mathcal{R}(\Psi)=\left\{p \in S\right.$; for all $\varepsilon, T>0$ there is a periodic $(\varepsilon, T)$-chain with $\left.p=p_{0}\right\}$. The restricted flow $\Psi \mid \mathcal{R}(\Psi)$ is chain recurrent, which is equivalent to the fact that $\mathcal{R}(\Psi)=A \cup A^{*}$ for every attractor-repeller pair $\left(A, A^{*}\right)$ of $\Psi \mid \mathcal{R}(\Psi)$. A chain recurrent flow on a connected space is chain transitive. Hence $\Psi$ restricted to a connected component $\mathcal{M}$ of $\mathcal{R}(\Psi)$ is chain transitive. The number of these connected components is finite iff there exists a finest Morse decomposition $\left\{\mathcal{M}_{1}, \ldots, \mathcal{M}_{m}\right\}$; in this case the Morse sets $\mathcal{M}_{i}$ coincide with the connected components of $\mathcal{R}(\Psi)$.

We use the standard definition of (real) vector bundles $\pi: E \rightarrow S$, as given e.g. in [Ka], Chapter I: $\pi$ is a continuous surjection such that the fibers $E_{p}:=$ $\pi^{-1}(p), p \in S$, are $d$-dimensional (real) vector spaces and $E$ is locally isomorphic to $S \times \mathbb{R}^{d}$. Our base space $S$ will be a compact, connected metric space. Furthermore, we keep fixed a (Riemannian) metric on $E$, constructed by adding up the inner products obtained from a finite coordinate covering of $E$ (cf. [Ka], Theorem I.8.7, or [SZ], Appendix). Note that any two metrics on $E$ are isomorphic (cf. [Ka], Theorem I.8.8 and Corollary I.8.9). We denote the inner product by $\langle\cdot, \cdot\rangle$, and the metric by $|\cdot|$.

The zero section $Z$ in $E$ is a continuous map $Z: S \rightarrow E$ given by $Z(p)=0 \in E_{p}$. For the following construction compare e.g. [SZ], Appendix. The projective bundle $\mathbb{P} E$ is given by $\mathbb{P} E=(E \backslash Z) / \sim$, where $e \sim e^{\prime}$ if $\pi(e)=\pi\left(e^{\prime}\right)$ and there exists $\alpha \in \mathbb{R}$ with $e=\alpha e^{\prime}$. The canonical projection map will be denoted by $\mathbb{P}: E \backslash Z \rightarrow \mathbb{P} E$. For $A \subset E$ we write $\mathbb{P} A=\{\mathbb{P} e ; e \in A \backslash Z\}$. There exists a unique projection $\mathbb{P} \pi: \mathbb{P} E \rightarrow S$ such that the following diagram commutes

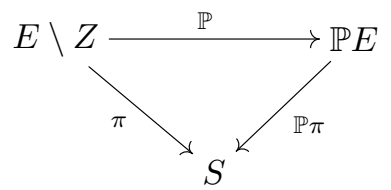

Note that $\mathbb{P} E$ is a compact metric space, iff $S$ is compact.

A linear flow $\Phi$ on a vector bundle $\pi: E \rightarrow S$ is a flow on $E$ preserving fibers such that

$$
\begin{aligned}
\Phi\left(t, e_{1}+e_{2}\right) & =\Phi\left(t, e_{1}\right)+\Phi\left(t, e_{2}\right), \quad t \in \mathbb{R}, e_{1}, e_{2} \in E_{p}, \text { and } \\
\Phi(t, \alpha e) & =\alpha \Phi(t, e), \quad t \in \mathbb{R}, \alpha \in \mathbb{R}, e \in E_{p},
\end{aligned}
$$


i.e. the induced maps $\Phi(t, \cdot)_{p}: E_{p} \rightarrow E_{\pi(t, e)}$ are linear. $\Phi$ induces a flow $\pi \Phi$ on the base space $S$, which we denote by $p \cdot t$ for $t \in \mathbb{R}, p \in S$, and a flow $\mathbb{P} \Phi$ on $\mathbb{E}$, see [SZ], Lemma 2.1.

A cohomology $F$ between a linear flow $\Phi$ on $\pi_{1}: E_{1} \rightarrow S$ and a linear flow $\Psi$ on $\pi_{2}: E_{2} \rightarrow S$ is a fiber respecting homeomorphism $F: E_{1} \rightarrow E_{2}$ such that the induced maps $F_{p}$ on the fibers are linear, and the diagram

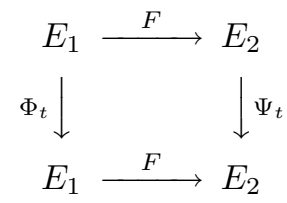

commutes. A cohomology $F$ between $\Phi$ and $\Psi$ induces a homeomorphism $\mathbb{P} F$ on the associated projective bundles such that

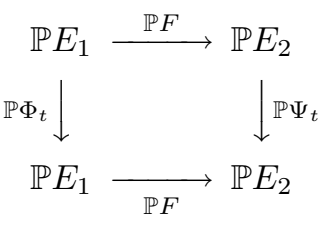

commutes for all $t \in \mathbb{R}$.

A linear flow $\Phi$ on a trivial bundle $S \times \mathbb{R}^{d}$ is called smooth if there exists a continuous function $A: S \rightarrow g \ell(d, \mathbb{R})$ such that the $\mathbb{R}^{d}$-component satisfies for all $t \in \mathbb{R}$

$$
\frac{d}{d t} \Phi(t, p) x=A(p \cdot t) \Phi(t, p) x, \quad(p, x) \in S \times \mathbb{R}^{d} .
$$

Here we have written $\Phi(t, p) x$ instead of $\Phi(t,(p, x))$ to emphasize that (2.2) concerns the $\mathbb{R}^{d}$-component of the flow, i.e. the associated cocycle. The following results will be needed in Section 4.

2.1. Lemma. Let $\Phi$ be a smooth linear flow on $S \times \mathbb{R}^{d}$ with $A(p)$ given in (2.2). Then

$$
\log |\Phi(t, p) x|-\log |x|=\int_{0}^{t}\langle A(p \cdot \tau) x, x\rangle d \tau
$$

for $t \in \mathbb{R},(p, x) \in S \times \mathbb{R}^{d}$. Here $\langle\cdot, \cdot\rangle$ and $|\cdot|$ denote the inner product, and the corresponding metric in $\mathbb{R}^{d}$.

This result follows easily by differentiating $|\Phi(t, p) x|^{2}$. For future use we define the continuous function

$$
q: S \times \mathbb{P}^{d-1} \rightarrow \mathbb{R}, q(p, x)=\langle A(p) x, x\rangle .
$$

$\left(\mathbb{P}^{d-1}\right.$ is the projective space in $\mathbb{R}^{d}$.)

2.2. Lemma. Let $\Phi$ be a linear flow on $S \times \mathbb{R}^{d}$. Then $\Phi$ is cohomologous to a smooth linear flow $\Psi$ on $S \times \mathbb{R}^{d}$.

For a proof see e.g. [EJ], Lemma 1.21 (smoothing lemma) or [JPS], Lemma 3.2. 


\section{The Morse Spectrum}

In this section we introduce the Morse spectrum of linear flows on vector bundles. It is based on Morse decompositions of the flow on the projective bundle, and consists of limits of (finite time) exponential growth rates of $(\varepsilon, T)$-chains. The Morse spectrum is the union of (not necessarily disjoint) intervals, and contains the Lyapunov spectrum of the flow. In fact, as we will see in Section 4, the boundary points of the intervals are actually Lyapunov exponents.

First of all, we summarize some results on the form of the chain recurrent components of the projected flow $\mathbb{P} \Phi$ and their relation to the chain recurrent components of the induced flow on the base space $S$.

3.1. Theorem. Let $\Phi$ be a linear flow on a vector bundle $\pi: E \rightarrow S$ with projected flow $\mathbb{P} \Phi$ on $\mathbb{P} \pi: \mathbb{P} E \rightarrow S$. Let $M \subset S$ be a chain recurrent component of the induced flow on $S$.

(i) The chain recurrent set of $\mathbb{P} \Phi \mid(\mathbb{P} \pi)^{-1} M$ has finitely many components (Morse sets) $\mathcal{M}_{1}, \ldots, \mathcal{M}_{\ell}$ with $1 \leq \ell=\ell(M) \leq d:=\operatorname{dim} E_{p}, p \in S$.

(ii) Every $\mathcal{M}_{i}$ defines a (continuous, constant dimensional) subbundle of $\pi^{-1} M$ via

$$
\mathcal{V}_{i}:=\left\{e \in \pi^{-1} M ; e \notin Z \Rightarrow \mathbb{P} e \in \mathcal{M}_{i}\right\}
$$

and the following decomposition into a Whitney sum holds

$$
\pi^{-1} M=\mathcal{V}_{1} \oplus \cdots \oplus \mathcal{V}_{\ell}
$$

(iii) Conversely, every chain recurrent component $\mathcal{M}$ of $\mathbb{P} \Phi$ is of the form described in (ii), in particular $\mathbb{P} \pi(\mathcal{M})$ is a chain recurrent component in $S$.

(iv) The chain recurrent sets $\mathcal{R}(\mathbb{P} \Phi)$ and $\mathcal{R}(\pi \Phi)$ satisfy

$$
\mathcal{R}(\mathbb{P} \Phi)=\mathbb{P}\left(\pi^{-1}(\mathcal{R}(\pi \Phi))\right) .
$$

Proof. Let $M$ be a chain recurrent component of the flow on the base space $S$. Note that $\pi \mid \pi^{-1} M: \pi^{-1} M \rightarrow S$ is a vector bundle (local trivialization is obtained by restricting the charts of $\pi: E \rightarrow S$ ), and the induced flow on $S$ restricted to $M$ is chain recurrent. Now (i) and (ii) follow from [Sg], Theorem 9.7, or [CK4], Theorem 4.5 .

Conversely, let $\mathcal{M}$ be a chain recurrent component of $\mathbb{P} \Phi$. Then $\mathbb{P} \pi(\mathcal{M})$ is a chain transitive set in $S$. It remains to prove maximality. Let $M \subset S$ be the chain recurrent component containing $\mathbb{P} \pi(\mathcal{M})$. Now apply the first part of the theorem to $M$ : It follows that $\mathcal{M}$ is contained in a chain recurrent component $\mathcal{M}_{i}$ of $\mathbb{P} \Phi \mid(\mathbb{P} \pi)^{-1} M$ with $\mathbb{P} \pi\left(\mathcal{M}_{i}\right)=M$ by the subbundle property of $\mathcal{V}_{i}$. From maximality of $\mathcal{M}$, it follows that $\mathcal{M}=\mathcal{M}_{i}$, proving (iii).

Finally, (3.3) follows from (i) and (iii).

The Morse spectrum of a linear flow $\Phi$ will be defined as the limits of (finite time) exponential growth rates of $(\varepsilon, T)$-chains in the chain recurrent components of $\mathbb{P} \Phi$ : Let $\Phi: \mathbb{R} \times E \rightarrow E$ be a linear flow on a vector bundle $\pi: E \rightarrow S$. For $\varepsilon, T>0$ an $(\varepsilon, T)$-chain $\zeta$ of $\mathbb{P} \Phi$ is given by $n \in \mathbb{N}, T_{0}, \ldots, T_{n-1} \geq T, \mathbb{P} e_{0}, \ldots, \mathbb{P} e_{n} \in \mathbb{P} E$ with $d\left(\mathbb{P} \Phi\left(T_{i}, \mathbb{P} e_{i}\right), \mathbb{P} e_{i+1}\right)<\varepsilon$ for $i=0 \ldots n-1$. (Here $d(\cdot, \cdot)$ is the induced metric on $\mathbb{P} E$.) Define the exponential growth rate of $\zeta$ by

$$
\lambda(\zeta)=\left(\sum_{i=0}^{n-1} T_{i}\right)^{-1} \sum_{i=0}^{n-1}\left(\log \left|\Phi\left(T_{i}, e_{i}\right)\right|-\log \left|e_{i}\right|\right)
$$


with $e_{i} \in \mathbb{P}^{-1}\left(\mathbb{P} e_{i}\right)$. Recall that $|\cdot|$ is a (fixed) metric on $E$.

3.2. Definition. Let $\Phi$ be a linear flow on a vector bundle. Define the Morse spectrum of $\Phi$ on a chain recurrent component $\mathcal{M}$ of the projected flow $\mathbb{P} \Phi$ as

$$
\begin{aligned}
\Sigma_{M o}(\mathcal{M}, \Phi)= & \left\{\lambda \in \mathbb{R} ; \text { there are } \varepsilon^{k} \rightarrow 0, T^{k} \rightarrow \infty \text { and }\left(\varepsilon^{k}, T^{k}\right) \text {-chains } \zeta^{k}\right. \text { in } \\
& \left.\mathcal{M} \text { with } \lambda\left(\zeta^{k}\right) \rightarrow \lambda \text { as } k \rightarrow \infty\right\} .
\end{aligned}
$$

The Morse spectrum of $\Phi($ on $\pi: E \rightarrow S)$ is

$$
\Sigma_{M o}(\Phi)=\cup\left\{\Sigma_{M o}(\mathcal{M}, \Phi) ; \mathcal{M} \text { is a chain recurrent component of } \mathbb{P} \Phi\right\} .
$$

For a compact invariant set $\mathcal{T} \subset \mathbb{P} E$ we define the Morse spectrum $\Sigma_{M o}(\mathcal{T}, \Phi)$ of $\Phi$ on $\mathcal{T}$ as the union of the Morse spectra of the chain recurrent components of $\mathbb{P} \Phi \mid \mathcal{T}$ defined as above.

The following lemma collects some simple facts about the Morse spectrum.

3.3. Lemma. Let $\Phi$ be a linear flow on a vector bundle $\pi: E \rightarrow S$. Then

(i) $\Sigma_{M o}(\Phi)=\Sigma_{M o}\left(\Phi \mid \pi^{-1}(\mathcal{R}(\pi \Phi))\right)$,

(ii) $\Sigma_{M o}(\Phi)=\bigcup\left\{\Sigma_{M o}\left(\Phi \mid \pi^{-1} M\right) ; M\right.$ is a chain recurrent component of $\left.\pi \Phi\right\}$.

(iii) If $\left\{M_{1} \ldots M_{n}\right\}$ is a Morse decomposition of the base flow $\pi \Phi$, then $\Sigma_{M o}(\Phi)=$ $\bigcup_{j=1}^{n} \Sigma_{M o}\left(\Phi \mid \pi^{-1} M_{j}\right)$, in particular, if $\pi \Phi$ has a finest Morse decomposition $\left\{M_{1}, \ldots, M_{n}\right\}$, then $\Sigma_{M o}(\Phi)=\bigcup_{j=1}^{n} \bigcup_{i=1}^{\ell\left(M_{j}\right)} \Sigma_{M o}\left(\mathcal{M}_{j i}, \Phi\right)$. Here $\mathcal{M}_{j i}, i=1$, $\ldots, \ell\left(M_{j}\right)$, denote the chain recurrent components of $\mathbb{P} \Phi \mid(\mathbb{P} \pi)^{-1} M_{j}$.

Proof. Part (i) follows from Theorem 3.1(iv), while (ii) and (iii) are immediate consequences of the definitions and of Theorem 3.1(i) and (iii).

For the rest of this section we will work with the periodic Morse spectrum, which has nice regularity properties. Recall that the periodic part of the Lyapunov spectrum are the Floquet exponents, and these are a part of the regular Lyapunov spectrum. It is an open question, under which conditions the Lyapunov spectrum agrees with (the closure of) the Floquet spectrum (see [CK6] for a result in this direction on $L^{\infty}$-families of time varying matrices). However, it will be shown in Section 4. that the Morse spectrum and the periodic Morse spectrum always agree.

3.4. Definition. Let $\Phi$ be a linear flow on a vector bundle, and let $\mathcal{M}$ be a chain recurrent component of the projected flow $\mathbb{P} \Phi$. Define the periodic Morse spectrum of $\Phi$ on $\mathcal{M}$ as

$$
\begin{aligned}
\Sigma_{M o}^{p}(\mathcal{M}, \Phi)= & \left\{\lambda \in \mathbb{R} ; \text { there are } \varepsilon^{k} \rightarrow 0, T^{k} \rightarrow \infty \text { and periodic }\left(\varepsilon^{k}, T^{k}\right)\right. \text {-chains } \\
& \left.\zeta^{k} \text { in } \mathcal{M} \text { with } \lambda\left(\zeta^{k}\right) \rightarrow \lambda \text { as } k \rightarrow \infty\right\} .
\end{aligned}
$$

As in Definition 3.2. we define $\Sigma_{M o}^{p}(\Phi)$ and $\Sigma_{M o}^{p}(\mathcal{T}, \Phi)$.

Note that the results from Lemma 3.3. remain valid for the periodic Morse spectrum. The following result describes the behavior of the periodic Morse spectrum under time reversal.

3.5. Proposition. For a linear flow $\Phi$ on a vector bundle $\pi: E \rightarrow S$ let the corresponding time reversed flow $\Phi^{*}$ be given by

$$
\Phi^{*}(t, e):=\Phi(-t, e), \quad t \in \mathbb{R}, e \in E .
$$


Then we have $\mathcal{R}(\mathbb{P} \Phi)=\mathcal{R}\left(\mathbb{P} \Phi^{*}\right)$ and $\Sigma_{M o}^{p}(\Phi)=-\Sigma_{M o}^{p}\left(\Phi^{*}\right)$.

Proof. Recall that the chain recurrent set of a flow is the intersection over all $A \cup A^{*}$, where $A$ is an attractor and $A^{*}$ its complementary repeller [Cn], Section II.6.2. or [Ak]. Under time reversal $A$ and $A^{*}$ exchange their respective roles, and one obtains the first assertion. This follows also from the following more explicit construction, which we need for the second assertion:

Let $\mathcal{M}$ be a chain recurrent component of $\mathbb{P} \Phi$ (and of $\mathbb{P} \Phi^{*}$ ). For $\varepsilon, T>0$ let $\zeta$ be a periodic $(\varepsilon, T)$-chain of $\mathbb{P} \Phi$ in $\mathcal{M}$ given by $n \in \mathbb{N}, T_{0}, \ldots, T_{n-1} \geq T$ and $\mathbb{P} e_{0}, \ldots, \mathbb{P} e_{n} \in \mathbb{P} E$. An $(\varepsilon, T)$-chain $\zeta^{*}$ of $\mathbb{P} \Phi^{*}$ in $\mathcal{M}$ is obtained by 'going backwards': Let $T_{0}^{*}, \ldots, T_{n-1}^{*} \geq T$ and $\mathbb{P} e_{0}^{*}, \ldots, \mathbb{P} e_{n}^{*} \in \mathbb{P} E$ be defined by

$$
\begin{aligned}
T_{i}^{*} & :=T_{n-i-1}, \quad i=0, \ldots, n-1, \\
\mathbb{P} e_{i}^{*} & :=\mathbb{P} \Phi\left(T_{n-i-1}, \mathbb{P} e_{n-i-1}\right), \quad i=0, \ldots, n-1, \\
\mathbb{P} e_{n}^{*} & :=\mathbb{P} e_{0}^{*} .
\end{aligned}
$$

Then we have for $i=0 \ldots n-2$

$$
\begin{aligned}
& d\left(\mathbb{P} \Phi^{*}\left(T_{i}^{*}, \mathbb{P} e_{i}^{*}\right), \mathbb{P} e_{i+1}^{*}\right) \\
= & d\left(\mathbb{P} \Phi^{*}\left(T_{n-i-1}, \mathbb{P} \Phi\left(T_{n-i-1}, \mathbb{P} e_{n-i-1}\right)\right), \mathbb{P} \Phi\left(T_{n-i-2}, \mathbb{P} e_{n-i-2}\right)\right) \\
= & d\left(\mathbb{P} e_{n-i-1}, \mathbb{P} \Phi\left(T_{n-i-2}, \mathbb{P} e_{n-i-2}\right)\right)<\varepsilon
\end{aligned}
$$

and

$$
d\left(\mathbb{P} \Phi^{*}\left(T_{n-1}^{*}, \mathbb{P} e_{n-1}^{*}\right), \mathbb{P} e_{n}^{*}\right)=d\left(\mathbb{P} e_{0}, \mathbb{P} \Phi\left(t_{n-1}, e_{n-1}\right)\right)<\varepsilon .
$$

For the chain exponents we obtain

$$
\begin{aligned}
\lambda(\zeta) & =\left(\sum_{i=0}^{n} T_{i}\right)^{-1} \sum_{i=0}^{n-1}\left(\log \left|\Phi\left(T_{i}, e_{i}\right)\right|-\log \left|e_{i}\right|\right) \\
& =\left(\sum_{i=0}^{n-1} T_{i}^{*}\right)^{-1} \sum_{i=0}^{n-1}\left(\log \left|e_{n-i-1}^{*}\right|-\log \left|\Phi^{*}\left(T_{n-i-1}^{*}, e_{n-i-1}^{*}\right)\right|\right) \\
& =-\lambda\left(\zeta^{*}\right) .
\end{aligned}
$$

Next we show that the periodic chain spectrum over a chain transitive set in $\mathbb{P} E$ is an interval.

The proof is based on a 'mixing' of exponents near the extremal values of the spectrum.

3.6. Theorem. Let $\Phi$ be a linear flow on a vector bundle $\pi: E \rightarrow S$, and let $\mathcal{T} \subset \mathbb{P} E$ be closed and $\mathbb{P} \Phi$-invariant such that $\mathbb{P} \Phi \mid \mathcal{T}$ is chain transitive. Define

$$
\kappa_{p}^{*}(\mathcal{T}):=\inf \Sigma_{M o}^{p}(\mathcal{T}, \Phi), \kappa_{p}(\mathcal{T}):=\sup \Sigma_{M o}^{p}(\mathcal{T}, \Phi) .
$$

Then $\Sigma_{M o}^{p}(\mathcal{T}, \Phi)=\left[\kappa_{p}^{*}(\mathcal{T}), \kappa_{p}(\mathcal{T})\right]$.

Proof. It suffices to show that for all $\lambda \in\left[\kappa_{p}^{*}(\mathcal{T}), \kappa_{p}(\mathcal{T})\right]$, all $\delta>0$, and all $\varepsilon, T>0$ there is a periodic $(\varepsilon, T)$-chain $\zeta$ in $\mathcal{T}$ with

$$
|\lambda(\zeta)-\lambda|<\delta
$$

Then closedness of the Morse spectrum yields the result. Fix $\delta>0$ and $\varepsilon, T>0$. There are periodic $(\varepsilon, T)$-chains $\zeta^{*}$ and $\zeta$ in $\mathcal{T}$ with

$$
\lambda\left(\zeta^{*}\right)<\kappa_{p}^{*}(\mathcal{T})+\delta, \quad \lambda(\zeta)>\kappa_{p}(\mathcal{T})-\delta .
$$


Denote the initial points of $\zeta^{*}$ and $\zeta$ by $\mathbb{P} e_{0}^{*}$ and $\mathbb{P} e_{0}$, respectively.

By chain transitivity there are $(\varepsilon, T)$-chains $\zeta_{1}$ from $\mathbb{P} e_{0}^{*}$ to $\mathbb{P} e_{0}$ and $\zeta_{2}$ from $\mathbb{P} e_{0}$ to $\mathbb{P} e_{0}^{*}$, both in $\mathcal{T}$. For $k \in \mathbb{N}$ let $\zeta^{* k}$ and $\zeta^{k}$ be the $k$-fold concatenation of $\zeta^{*}$, and of $\zeta$, respectively. Then for $k, \ell \in \mathbb{N}$ the concatenation $\zeta^{k, \ell}=\zeta_{2} \circ \zeta^{k} \circ \zeta_{1} \circ \zeta^{* \ell}$ is a periodic $(\varepsilon, T)$-chain in $\mathcal{T}$. Note that the exponents of concatenated chains are convex combinations of the corresponding exponents. Hence for every $\lambda \in\left[\lambda\left(\zeta^{*}\right), \lambda(\zeta)\right]$ one finds numbers $k, \ell \in \mathbb{N}$ such that $\left|\lambda\left(\zeta^{k, \ell}\right)-\lambda\right|<\delta$. This proves (3.7).

The next result shows that the periodic Morse spectrum contains the entire Lyapunov spectrum of the flow $\Phi$. The relation of the Morse spectrum to other spectral concepts will be discussed in Section 5. Recall the definition of a Lyapunov exponent

$$
\lambda(e):=\limsup _{t \rightarrow \infty} \frac{1}{t} \log |\Phi(t, e)| .
$$

The Lyapunov spectrum of a linear flow $\Phi$ on a vector bundle $\pi: E \rightarrow S$ is defined as

$$
\Sigma_{L y}(\Phi):=\{\lambda(e) ; e \in E\}
$$

3.7. Theorem. Let $\Phi$ be a linear flow on a vector bundle $\pi: E \rightarrow S$. Then for all $e \in E$ we have

$$
\lambda(e) \in \Sigma_{M o}^{p}(\omega(\mathbb{P} e), \Phi) \subset \Sigma_{M o}^{p}(\mathcal{M}, \Phi),
$$

where $\mathcal{M}$ is the chain recurrent component of $\mathbb{P} \Phi$ containing $\omega(\mathbb{P e} e) \subset \mathbb{P} E$. In particular

$$
\Sigma_{L y}(\Phi) \subset \Sigma_{M o}^{p}(\Phi)
$$

Proof. The inclusion (3.11) is a direct consequence of (3.10). In order to show (3.10) recall that $\omega$-limits sets are connected and contained in the chain recurrent set. Hence the component $\mathcal{M}$ containing $\omega(\mathbb{P} e)$ is well defined for $e \in E$. We have to show the following: For all $\delta>0$, and all $\varepsilon, T>0$ there exists a periodic $(\varepsilon, T)$-chain $\zeta$ in $\omega(\mathbb{P} e)$ with

$$
|\lambda(e)-\lambda(\zeta)|<\delta
$$

Fix $\delta>0, \varepsilon>0, T>1$. Since $\mathbb{P} \Phi$ is uniformly continuous on the compact set $[0,2 T] \times \mathbb{P} E$, there is $\delta_{1}=\delta_{1}(\delta, \varepsilon, T)>0$ such that for all $\mathbb{P} a, \mathbb{P} b \in \mathbb{P} E$ with $d(\mathbb{P} a, \mathbb{P} b)<\delta_{1}$ it follows that

$$
d(\mathbb{P} \Phi(t, \mathbb{P} a), \mathbb{P} \Phi(t, \mathbb{P} b))<\frac{\varepsilon}{3}
$$

and

$$
|\log | \Phi(t, a)|-\log | \Phi(t, b)||<\frac{\delta}{4}
$$

for all $t \in[0,2 T]$, where $a \in \mathbb{P}^{-1}(\mathbb{P} a), b \in \mathbb{P}^{-1}(\mathbb{P} b)$ are chosen appropriately with $|a|=|b|=1$. We may assume that $|e|=1$ and that

$$
d(\mathbb{P} \Phi(t, \mathbb{P} e), \omega(\mathbb{P} e))<\delta_{1} \text { for all } t>0,
$$

replacing, if necessary, $e$ by $|\Phi(\tau, e)|^{-1} \Phi(\tau, e)$ for $\tau$ large enough. By definition of the Lyapunov exponent there are $t_{n} \rightarrow \infty$ with

$$
\lambda(e)=\lim _{n \rightarrow \infty} \frac{1}{t_{n}} \log \left|\Phi\left(t_{n}, e\right)\right|,
$$


where we can assume without loss of generality that $\mathbb{P} \Phi\left(t_{n}, e\right)$ converges for $t_{n} \rightarrow \infty$. Now fix $N \in \mathbb{N}$ such that for all $n \geq N$

$$
d\left(\mathbb{P} \Phi\left(t_{n}, e\right), \mathbb{P} \Phi\left(t_{N}, e\right)\right)<\frac{\varepsilon}{3} .
$$

Setting $\sigma_{n}=t_{n}-t_{N}$ we have

$$
\lambda(e)=\lambda\left(\Phi\left(t_{N}, e\right)\right)=\lim _{n \rightarrow \infty} \frac{1}{\sigma_{n}} \log \left|\Phi\left(\sigma_{n}, \Phi\left(t_{N}, e\right)\right)\right| .
$$

Choose $n$ large enough such that with $T_{0}:=\sigma_{n}$ we have $T_{0}>2 T$ and

$$
\left|\lambda(e)-\frac{1}{T_{0}} \log \right| \Phi\left(T_{0}, \Phi\left(t_{N}, e\right)\right)||<\frac{\delta}{2} .
$$

Clearly, (3.15) remains valid with $\Phi\left(t_{N}, e\right)$ instead of $e$. Hence writing $e$ instead of $\Phi\left(t_{N}, e\right)$ in (3.16) and (3.17), we obtain in addition to (3.15)

$$
\begin{gathered}
d\left(\mathbb{P} \Phi\left(T_{0}, \mathbb{P} e\right), \mathbb{P} e\right)<\frac{\varepsilon}{3}, \text { and } \\
\left|\lambda(e)-\frac{1}{T_{0}} \log \right| \Phi\left(T_{0}, e\right)||<\frac{\delta}{2} .
\end{gathered}
$$

We partition the interval $\left[0, T_{0}\right]$ into pieces of length $\tau_{j}$ with $T \leq \tau_{j}<2 T$ with $j=0, \ldots, \ell-1$. Thus

$$
T_{0}=\sum_{j=0}^{\ell-1} \tau_{j} \quad \text { and } \quad T_{0}>\ell .
$$

Set $y_{0}=e, y_{j+1}=\Phi\left(\tau_{j}, y_{j}\right)$ for $j=0, \ldots, \ell-1$. Then $\Phi\left(T_{0}, e\right)=y_{\ell}$ and

$$
\begin{aligned}
\frac{1}{T_{0}} \log \left|\Phi\left(T_{0}, e\right)\right| & =\frac{1}{T_{0}} \sum_{j=0}^{\ell-1}\left(\log \left|y_{j+1}\right|-\log \left|y_{j}\right|\right) \\
& =\left(\sum_{j=0}^{\ell-1} \tau_{j}\right)^{-1} \sum_{j=0}^{\ell-1}\left(\log \left|\Phi\left(\tau_{j}, y_{j}\right)\right|-\log \left|y_{i}\right|\right) .
\end{aligned}
$$

Define an $\left(\frac{\varepsilon}{3}, T\right)$-chain $\tilde{\zeta}$ in $\mathbb{P} E$ by $\ell \in \mathbb{N}, \tau_{0}, \ldots, \tau_{\ell-1} \geq T, \mathbb{P} y_{0}, \ldots, \mathbb{P} y_{\ell-1}, \mathbb{P} y_{0} \in$ $\mathbb{P} E$, noting that by (3.18) we have

$$
d\left(\mathbb{P} \Phi\left(\tau_{\ell-1}, \mathbb{P} y_{\ell-1}\right), \mathbb{P} y_{0}\right)<\frac{\varepsilon}{3} .
$$

Using (3.21) we obtain

$$
\lambda(\tilde{\zeta})=\frac{1}{T_{0}} \log \left|\Phi\left(T_{0}, e\right)\right|
$$

But the chain $\tilde{\zeta}$ is not necessarily contained in $\omega(\mathbb{P} e)$. In order to obtain an appropriate chain $\zeta$ in $\omega(\mathbb{P} e)$, we use (3.13) and (3.15): For $\mathbb{P} y_{j}=\mathbb{P} \Phi\left(\tau_{j}, \mathbb{P} y_{j-1}\right) \quad(j=$ $0 \ldots \ell-1)$ we find points $\mathbb{P} z_{0}, \ldots, \mathbb{P} z_{\ell-1}, \mathbb{P} z_{\ell}=\mathbb{P} z_{0}$ in $\omega(\mathbb{P} e)$ with

$$
d\left(\mathbb{P} \Phi\left(t, \mathbb{P} y_{j}\right), \mathbb{P} \Phi\left(t, \mathbb{P} z_{j}\right)\right)<\frac{\varepsilon}{3} \text { for } t \in[0,2 T] .
$$


Hence we obtain for $j=0, \ldots, \ell-1$

$$
\begin{aligned}
d(\mathbb{P} \Phi & \left.\left(\tau_{j}, \mathbb{P} z_{j}\right), \mathbb{P} z_{j+1}\right) \leq d\left(\mathbb{P} \Phi\left(t_{j}, \mathbb{P} z_{j}\right), \mathbb{P} \Phi\left(\tau_{j}, \mathbb{P} y_{j}\right)\right) \\
& +d\left(\mathbb{P} \Phi\left(\tau_{j}, \mathbb{P} y_{j}\right), \mathbb{P} y_{j+1}\right)+d\left(\mathbb{P} y_{j+1}, \mathbb{P} z_{j+1}\right) \\
& <\frac{\varepsilon}{3}+\frac{\varepsilon}{3}+\frac{\varepsilon}{3}=\varepsilon
\end{aligned}
$$

where for $j=\ell-1$ we have used (3.22). We have shown that $\ell \in \mathbb{N}, \tau_{1}, \ldots, \tau_{\ell-1} \geq T$, and $\mathbb{P} z_{0}, \ldots, \mathbb{P} z_{\ell-1}, \mathbb{P} z_{\ell}=\mathbb{P} z_{0} \in \mathbb{P} E$ define a periodic $(\varepsilon, T)$-chain in $\omega(\mathbb{P} e)$.

For the exponential growth rate of the chain $\zeta(3.19)$ and (3.23) yield

$$
\begin{aligned}
\mid \lambda(e) & -\lambda(\zeta)|\leq| \lambda(e)-\frac{1}{T_{0}} \log \left|\Phi\left(T_{0}, e\right)\right||+| \lambda(\tilde{\zeta})-\lambda(\zeta) \mid \\
& <\frac{\delta}{2}+\frac{1}{T_{0}} \sum_{j=o}^{\ell-1}\left\{\log \left|\Phi\left(\tau_{j}, y_{j}\right)\right|-\log \left|y_{j}\right|-\left[\log \left|\Phi\left(t_{j}, z_{j}\right)\right|-\log \left|z_{j}\right|\right]\right\} .
\end{aligned}
$$

Here we may take $y_{j} \in \mathbb{P}^{-1}\left(\mathbb{P} y_{i}\right)$ and $z_{j} \in \mathbb{P}^{-1}\left(\mathbb{P} z_{j}\right)$ appropriately to obtain from (3.14), (3.15) and (3.20)

$$
|\lambda(e)-\lambda(\zeta)|<\frac{\delta}{2}+\frac{1}{T_{0}} \ell \frac{\delta}{2}<\delta
$$

This proves (3.12) and concludes the proof of the theorem.

3.8. Remark. A flow restricted to an $\omega$-limit set is chain transitive, see e.g. [Cn], Section II.6.3.C. Hence combining Theorems 3.6 and 3.7 we obtain for all $e \in E$

$$
\lambda(e) \in\left[\kappa_{p}^{*}(\omega(\mathbb{P} e)), \kappa_{p}(\omega(\mathbb{P} e))\right]
$$

In fact, the proof of Theorem 3.7 shows that all limit points of $\frac{1}{t} \log |\Phi(t, e)|$ for $t \rightarrow \infty$ are contained in $\left[\kappa_{p}^{*}(\omega(\mathbb{P} e)), \kappa_{p}(\omega(\mathbb{P} e))\right]$.

3.9. Remark. The result (3.10) remains true for linear flows on vector bundles over a noncompact base space $S$, provided that the positive orbit $\{\Phi(t, e) ; t \geq 0\}$ is relatively compact.

3.10. Remark. Let $\lambda^{-}(e):=\limsup _{t \rightarrow-\infty} \frac{1}{t} \log |\Phi(t, e)|$ be the backward Lyapunov exponent of $e \in E$ under the flow $\Phi$. Then $\lambda^{-}(e) \in \Sigma_{M o}^{p}\left(\Phi \mid \mathbb{P}^{-1}\left(\omega^{*}(\mathbb{P} e)\right) \subset \Sigma_{M o}^{p}\left(\mathcal{M}^{*}, \Phi\right)\right.$, where $\mathcal{M}^{*}$ is the chain recurrent component of $\mathbb{P} \Phi$ containing $\omega^{*}(\mathbb{P} e)$. This is proved in exactly the same way as Theorem 3.7, using the periodicity of the approximating chains.

The rest of this section is concerned with the behavior of the Morse spectrum under perturbations of the flow. In general, continuity of the spectrum cannot be expected, since it does not even hold for the chain recurrent set. However, a certain upper semicontinuity property can be established.

We consider the following family $\Phi^{\alpha}$ of linear flows on a vector bundle $\pi: E \rightarrow S$, parametrized by $\alpha \in A$, where $A$ is a compact metric space: Let $\Phi: A \times \mathbb{R} \times E \rightarrow E$ be a continuous map such that for all $\alpha \in A$

$$
\Phi^{\alpha}:=\Phi(\alpha, \cdot, \cdot): \mathbb{R} \times E \rightarrow E
$$

is a linear flow on $\pi: E \rightarrow S$. 
3.11. Theorem. Consider the family (3.25) of parametrized linear flows. Set $\Phi^{0}:=\Phi^{\alpha^{0}}$ for some $\alpha^{0} \in A$, and let $\mathcal{T}^{0} \subset \mathbb{P} E$ be a compact, $\mathbb{P} \Phi^{0}$-invariant set such that $\mathbb{P} \Phi^{0} \mid \mathcal{T}^{0}$ is chain transitive. Assume that for every neighborhood $\mathcal{W}$ of $\mathcal{T}^{0}$ there exists a neighborhood $V$ of $\alpha^{0}$ in $A$ such that for all $\alpha \in V$ there is a compact, $\mathbb{P} \Phi^{\alpha}$-invariant set $\mathcal{T}^{\alpha}$ with $\mathcal{T}^{\alpha} \subset \mathcal{W}$. Then

$$
\begin{aligned}
& \left\{\lambda \in \mathbb{R} ; \text { there are } \alpha^{k} \rightarrow \alpha^{0} \text { and } \lambda^{k} \in \Sigma_{M o}^{p}\left(\mathcal{T}^{\alpha^{k}}, \Phi^{\alpha^{k}}\right) \text { with } \lambda^{k} \rightarrow \lambda\right\} \\
& \subset \Sigma_{M o}^{p}\left(\mathcal{T}^{0}, \Phi^{0} \mid \mathbb{P}^{-1}\left(\mathcal{T}^{0}\right)\right) .
\end{aligned}
$$

Proof. Let $\lambda$ be in the set on the left hand side of (3.26), with corresponding $\alpha^{k}$ and $\mathcal{T}^{k}:=\mathcal{T}^{\alpha^{k}}$. It suffices to show that for every $\delta>0$ and all $\varepsilon, T>0$ there is a periodic $(\varepsilon, T)$-chain $\zeta$ of $\mathbb{P} \Phi^{0}$ in $\mathcal{T}^{0}$ with

$$
|\lambda-\lambda(\zeta)|<\delta
$$

Choose $\delta>0, \varepsilon>0$ and $T>1$. Then there is $k_{0}:=k_{0}(\delta, \varepsilon, T) \in \mathbb{N}$ such that for all $k \geq k_{0}$ the following holds: For all $\mathbb{P} a \in \mathcal{T}^{k}$ there exists $\mathbb{P} b \in \mathcal{T}^{0}$ such that for all $t \in[0,2 T]$

$$
d\left(\mathbb{P} \Phi^{k}(t, \mathbb{P} a), \mathbb{P} \Phi^{0}(t, \mathbb{P} b)\right)<\frac{\varepsilon}{3},
$$

where we have set $\Phi^{k}:=\Phi^{\alpha^{k}}$. This estimate follows directly from the uniform continuity of $\mathbb{P} \Phi \mid A \times[0,2 T] \times \mathbb{P} E$ and the assumptions on $\left\{\mathcal{T}^{\alpha}, \alpha \in A\right\}$. Similarly, we may choose $k_{0}$ large enough such that for all $k \geq k_{0}$

$$
|\log | \Phi^{k}(t, a)|-\log | \Phi^{0}(t, b)||<\frac{\delta}{4}
$$

for an appropriate choice of $a \in \mathbb{P}^{-1}(\mathbb{P} a)$ and $b \in \mathbb{P}^{-1}(\mathbb{P} b)$. From the definition of $\lambda$ it follows that there are, for $k$ large enough, periodic $\left(\frac{\varepsilon}{3}, T\right)$ - chains $\zeta^{k}$ of $\mathbb{P} \Phi^{k} \mid \mathcal{T}^{k}$, given by $n^{k} \in \mathbb{N}, T_{0}^{k}, \ldots, T_{n_{k}}^{k} \geq T, \mathbb{P} y_{0}^{k} \ldots \mathbb{P} y_{n^{k}}^{k} \in \mathcal{T}^{k}$ with

$$
\begin{gathered}
d\left(\mathbb{P} \Phi^{k}\left(T_{j}^{k}, \mathbb{P} y_{j}^{k}\right), \mathbb{P} y_{j+1}^{k}\right)<\frac{\varepsilon}{3} \text { for } j=0 \ldots n^{k}-1, \\
\left|\lambda-\lambda\left(\zeta^{k}\right)\right|<\frac{\delta}{2} .
\end{gathered}
$$

Fix $k$ and repeat the construction in the proof of Theorem 3.7 for each interval $\left[0, T_{j}^{k}\right], j=0 \ldots n_{k}-1$ : Partition the intervals $\left[0, T_{j}^{k}\right]$ into pieces of length $\tau_{j i}^{k}$ with $T \leq \tau_{j i}^{k}<2 T$. Then use (3.28)-(3.30) to find elements $\mathbb{P} z_{j i}^{k} \in \mathcal{T}^{0}$, which yield a periodic $(\varepsilon, T)$-chain $\zeta^{0}$ of $\mathbb{P} \Phi^{0}$ such that

$$
\left|\lambda\left(\zeta^{k}\right)-\lambda\left(\zeta^{0}\right)\right|<\frac{\delta}{2}
$$

Now (3.31) and (3.32) yield the assertion (3.27).

It is well known (cf. e.g. [Si], Section 3.2.2) that the chain recurrent set depends upper semicontinuously on perturbations of the flow: This set cannot increase discontinuously under small perturbations, but it can decrease in jumps. (Actually, it is easy to find examples, in which a chain recurrent component vanishes under small perturbations: Take e.g. a family of one dimensional differential equations at a saddle-node bifurcation.) With this in mind, one can prove the following version of the preceding theorem. 
3.12. Corollary. Consider the family (3.25) of parametrized flows $\Phi^{\alpha}$ and set $\Phi^{k}:=\Phi^{\alpha^{k}}$ for a sequence $\alpha^{k} \rightarrow \alpha^{0}$ in A. Assume that the induced flow $\pi \Phi^{0}$ on the base space $S$ has a finest (finite) Morse decomposition. Then

$$
\left\{\lambda \in \mathbb{R} ; \text { there are } \lambda^{k} \in \Sigma_{M o}^{p}\left(\Phi^{k}\right) \text { with } \lambda^{k} \rightarrow \lambda\right\} \subset \Sigma_{M o}^{p}\left(\Phi^{0}\right) .
$$

Proof. By constructing appropriate $(\varepsilon, T)$-chains it is easy to see that $\mathcal{T}^{0}:=\{\mathbb{P} e \in$ $\mathbb{P} E$; there are $\mathbb{P} e^{k} \in \mathcal{R}\left(\mathbb{P} \Phi^{k}\right)$ with $\left.\mathbb{P} e^{k} \rightarrow \mathbb{P} e\right\} \subset \mathcal{R}\left(\mathbb{P} \Phi^{0}\right) . \quad \mathcal{T}^{0}$ is compact, and hence for every neighborhood $\mathcal{W}$ of $\mathcal{T}^{0}$ there exists $K \in \mathbb{N}$ such that for all $k \geq K$ we have $\mathcal{R}\left(\mathbb{P} \Phi^{k}\right) \subset \mathcal{W}$. By assumption, $\mathcal{R}\left(\pi \Phi^{0}\right)$ consists of finitely many components $M_{1}, \ldots, M_{n}$, and hence $\mathcal{R}\left(\mathbb{P} \Phi^{0}\right)$ has finitely many components $\mathcal{M}_{j i}, j=$ $1 \ldots n, i=1 \ldots \ell\left(M_{j}\right)$, compare Theorem 3.1. Therefore $\mathcal{W}$ can be chosen as $\mathcal{W}=\bigcup_{j=1}^{n} \bigcup_{i=1}^{\ell\left(M_{j}\right)} \mathcal{W}\left(\mathcal{M}_{j i}\right)$, where the $\mathcal{W}\left(\mathcal{M}_{j i}\right)$ have pairwise disjoint closures.

Now take $\lambda$ from the left hand side of (3.33). Then for each $k \in \mathbb{N}$ there exists a chain recurrent component $\mathcal{M}^{k}$ of $\mathbb{P} \Phi^{k}$ such that $\lambda^{k} \in \Sigma_{M o}^{p}\left(\mathcal{M}^{k}, \Phi^{k}\right)$. Hence there is a subsequence $\lambda^{k_{m}} \rightarrow \lambda$ such that the chains approximating the $\lambda^{k_{m}}, m \in \mathbb{N}$, are contained in one of the $\mathcal{W}\left(\mathcal{M}_{j i}\right)$. Now the proof proceeds as for Theorem 3.10 , replacing $\mathcal{T}^{k}$ by the corresponding chain recurrent components of $\mathbb{P} \Phi^{k_{m}}$ in $\mathcal{W}\left(\mathcal{M}_{j i}\right)$.

3.13. Remark. The last theorem may be viewed as a 'roughness' result for the Morse spectrum. This kind of result is well known for exponential dichotomies, cf. e.g. [MS], Section 8, [DK], Theorem IV. 5.1, [Cp], Lecture 4, or [SS2], Theorem 6 . The Lyapunov spectrum of a flow does, in general, not depend continuously on parameters, see e.g. [LY]. However, Theorem 3.11 and Corollary 3.12 show upper semicontinuity of the periodic Morse spectrum, which includes the Lyapunov spectrum by Theorem 3.7. In Section 6 we will discuss situations in which the Lyapunov and the Morse spectrum agree, and hence upper semicontinuity of the Lyapunov spectrum follows. Compare also [CK6] for continuity properties of both spectra for $L^{\infty}$-families of time varying matrices, and [AN] for related results concerning Lyapunov exponents of stochastic flows.

3.14. Remark. The proof of Corollary 3.12 will not yield continuity of the Morse spectrum, even if the chain recurrent components of the family $\mathbb{P} \Phi^{\alpha}$ depend continuously on $\alpha$. At this moment it is not clear to us, under which conditions the Morse spectrum does vary continuously with $\alpha$.

\section{Ergodic Theory of the Morse Spectrum}

The main result of this section is Theorem 4.6 which shows that the boundary points of the Morse spectrum are attained as Lyapunov exponents in the support of ergodic invariant measures. In particular, the Morse spectrum and the periodic Morse spectrum agree. For the proof of Theorem 4.6, we first reduce the problem to smooth linear flows on trivial vector bundles. A generalization of the Krylov-Bogolyubov construction of invariant measures to chains is then used for the development of ergodic theory for the Morse spectrum.

First of all, we analyze the behavior of the chain recurrent set and the Morse spectrum under cohomology.

4.1. Proposition. Let $F: E_{1} \rightarrow E_{2}$ be a cohomology between the linear flows $\Phi$ on $\pi_{1}: E_{1} \rightarrow S$ and $\Psi$ on $\pi_{2}: E_{2} \rightarrow S$. Then 
(i) $\mathbb{P} F(\mathcal{R}(\mathbb{P} \Phi))=\mathcal{R}(\mathbb{P} \Psi)$,

(ii) $\Sigma_{M o}(\Phi)=\Sigma_{M o}(\Psi), \Sigma_{M o}^{p}(\Phi)=\Sigma_{M o}^{p}(\Psi)$,

(iii) $\Sigma_{L y}(\Phi)=\Sigma_{L y}(\Psi)$.

Proof. (i) Note that $\mathbb{P} F: \mathbb{P} E_{1} \rightarrow \mathbb{P} E_{2}$ and $(\mathbb{P} F)^{-1}$ are uniformly continuous, since $\mathbb{P} E_{1}$ and $\mathbb{P} E_{2}$ are compact. Define for $\varepsilon>0$ the modulus of continuity $\gamma(\varepsilon):=$ $\sup \left\{\delta>0 ; d(\mathbb{P} a, \mathbb{P} b)<\delta\right.$ implies $d(\mathbb{P} F(\mathbb{P} a), \mathbb{P} F(\mathbb{P} b)) \leq \varepsilon$ for $\left.\mathbb{P} a, \mathbb{P} b \in \mathbb{P} E_{1}\right\}$. Then $\gamma(\varepsilon)>0$, and hence a $(\gamma(\varepsilon), T)$-chain for $\mathbb{P} \Phi$ is mapped under $\mathbb{P} F$ onto an $(\varepsilon, T)$ chain for $\mathbb{P} \Psi$. In particular, $\mathbb{P} F$ maps the chain recurrent components of $\mathbb{P} \Phi$ onto chain recurrent components of $\mathbb{P} \Psi$.

(ii) Let $\lambda \in \Sigma_{M o}(\mathcal{M}, \Phi)$ for some chain recurrent component $\mathcal{M}$ of $\mathbb{P} \Phi$, and fix $\delta>0$. It suffices to show that for all $\varepsilon, T>0$ there is an $(\varepsilon, T)$-chain $\zeta$ for $\mathbb{P} \Psi$ in $\mathbb{P} F(\mathcal{M})$ with

$$
\lambda(\zeta) \leq \lambda+\delta
$$

The operator norm of the fiber maps $F_{p}$ is bounded, say

$$
\left\|F_{p}\right\| \leq c \quad \text { for some } c>0 \text {. }
$$

Fix $\varepsilon, T>0$ with $\frac{1}{T} \log c<\frac{\delta}{2}$. As above, a $(\gamma(\varepsilon), T)$-chain $\tilde{\zeta}$ of $\mathbb{P} \Phi$ gives rise to an $(\varepsilon, T)$-chain $\zeta$ for $\mathbb{P} \Psi$, which can be described by $n \in \mathbb{N}, T_{0}, \ldots, T_{n-1} \geq T$, and $\mathbb{P} F\left(\mathbb{P} e_{0}\right), \ldots, \mathbb{P} F\left(\mathbb{P} e_{n}\right)$. We may choose $\tilde{\zeta}$ in $\mathcal{M}$ with

$$
\lambda(\tilde{\zeta}) \leq \lambda+\frac{\delta}{2},
$$

and obtain for $\lambda(\zeta)$ with $e_{i} \in \mathbb{P}^{-1}\left(\mathbb{P} e_{i}\right)$

$$
\lambda(\zeta)=\left(\sum_{i=0}^{n-1} T_{i}\right)^{-1} \sum_{i=0}^{n-1}\left(\log \left|\Psi\left(T_{i}, F e_{i}\right)\right|-\log \left|F e_{i}\right|\right) .
$$

Choose $e_{i}$ with $\left|F e_{i}\right|=1$. Then we obtain by cohomology using (4.2) and (4.3)

$$
\begin{aligned}
\lambda(\zeta) & =\left(\sum_{i=0}^{n-1} T_{i}\right)^{-1} \sum_{i=0}^{n-1} \log \left|F \Phi\left(T_{i}, e_{i}\right)\right| \\
& \leq\left(\sum_{i=0}^{n-1} T_{i}\right) \sum_{i=0}^{n-1}\left(\log \left\|F_{\pi e_{i}}\right\|+\log \left|\Phi\left(T_{i}, e_{i}\right)\right|\right) \\
& \leq \frac{\delta}{2}+\lambda(\tilde{\zeta}) \\
& \leq \lambda+\delta .
\end{aligned}
$$

Since $\mathbb{P} F$ maps periodic chains into periodic chains, this argument also proves the second part of (ii).

(iii) The well known assertion for the Lyapunov spectrum follows similarly, see e.g. [JPS].

Next we show that every linear flow on a vector bundle is cohomologous to a subflow of a smooth linear flow on a trivial vector bundle, and that the Morse spectrum is preserved under this cohomology. The proof is based on a construction due to [JPS], Lemma 3.4. 
4.2. Theorem. Let $\Phi$ be a linear flow on a vector bundle $\pi: E \rightarrow S$. Then there exists a smooth linear flow $\Psi$ on a trivial bundle $S \times \mathbb{R}^{m}$ for some $m \in \mathbb{N}$, and a decomposition $S \times \mathbb{R}^{m}=E^{\prime} \oplus E^{\prime \prime}$ into $\Psi$-invariant subbundles such that there exists a cohomology $F$ between $\Phi$ and $\Psi \mid E^{\prime}$, and the following holds:

$$
\begin{aligned}
\mathbb{P} F(\mathcal{R}(\mathbb{P} \Phi)) & =\mathcal{R}\left(\mathbb{P} \Psi \mid \mathbb{P} E^{\prime}\right), \\
\mathcal{R}(\mathbb{P} \Psi) & =\mathcal{R}\left(\mathbb{P} \Psi \mid \mathbb{P} E^{\prime}\right) \dot{\cup} \mathcal{R}\left(\mathbb{P} \Psi \mid \mathbb{P} E^{\prime \prime}\right), \\
\Sigma_{M o}(\Phi) & =\Sigma_{M o}\left(\Psi \mid E^{\prime}\right), \Sigma_{M o}^{p}(\Phi)=\Sigma_{M o}^{p}\left(\Psi \mid E^{\prime}\right), \\
\Sigma_{M o}(\Psi) & =\Sigma_{M o}\left(\Psi \mid E^{\prime}\right) \dot{\cup} \Sigma_{M o}\left(\Psi \mid E^{\prime \prime}\right), \\
\Sigma_{M o}^{p}(\Psi) & =\Sigma_{M o}^{p}\left(\Psi \mid E^{\prime}\right) \dot{\cup} \Sigma_{M o}^{p}\left(\Psi \mid E^{\prime \prime}\right) ;
\end{aligned}
$$

here $\dot{U}$ denotes disjoint union.

Proof. By a well known result in the theory of vector bundles (see e.g. [Ka], Theorem I.6.5) there exists $m \in \mathbb{N}$ and a subbundle decomposition $S \times \mathbb{R}^{m}=E_{1} \oplus E_{2}$ such that $E_{1}$ is isomorphic (as a vector bundle) to $E$. Hence there is an orthogonal projection $Q_{1}: S \times \mathbb{R}^{m}=E_{1} \oplus E_{2} \rightarrow E_{1}$, and an isomorphism $I_{1}: E \rightarrow E_{1}$ such that $E \stackrel{I_{1}}{\hookrightarrow} E_{1} \longrightarrow E_{1} \oplus E_{2} \stackrel{Q_{1}}{\longrightarrow} i m Q_{1}=E_{1}$ is an isomorphism, which we denote by $G_{1}$. $Q_{1}$ induces a continuous map (cocycle) $W_{1}: S \longrightarrow \mathfrak{g}(m, d)$ (the Grassmannian of $d$-dimensional subspaces in $\mathbb{R}^{m}$ ), defined by $W_{1}=Q_{1} \circ \pi^{-1}$. (Note that $W_{1}$ is well defined, although $\pi^{-1}$ is not a map.) Recall that $d=\operatorname{dim} E_{p}$.

By [JPS], Lemma 3.3 there exists a smooth map $W_{2}: S \longrightarrow \mathfrak{g}(m, d)$, uniformly close to $W_{1}$. Here smooth means that the map $p \mapsto \frac{d}{d t} W_{2}(p \cdot t)$ from $S$ into the tangent bundle of $\mathfrak{g}(m, d)$ exists and is continuous. Define $Q_{2}: E_{1} \oplus E_{2} \longrightarrow S \times \mathbb{R}^{m}$ such that $W_{2}=Q_{2} \circ \pi^{-1}$, and $Q_{2}^{\perp}:=I d-Q_{2}$. It follows that $E \stackrel{I_{1}}{\longrightarrow} E_{1} \hookrightarrow$ $E_{1} \oplus E_{2} \stackrel{Q_{2}}{\longrightarrow} i m Q_{2}$ is an isomorphism of vector bundles, which we call $G_{2}$.

Next we define a flow on $S \times \mathbb{R}^{m}$, which leaves $i m Q_{2}$ and $i m Q_{2}^{\perp}$ invariant. Set $W_{2}^{\prime}(p)=\frac{d}{d t} W_{2}(p \cdot t)(0)$, similarly for $W_{2}^{\perp^{\prime}}(p)$. Let

$$
X(p)=W_{2}^{\prime}(p) W_{2}(p)+W_{2}^{\perp^{\prime}}(p) W_{2}^{\perp}(p) \quad \text { for } p \in S
$$

and consider the fundamental solution $\Lambda_{1}(t, p)$ of $\quad \dot{x}=X(p \cdot t) x \quad$ for $x \in \mathbb{R}^{m}$ with $\Lambda_{1}(0, p)=I d$. Then (cf. [DK], Theorem IV. 1.1) we obtain

$$
\begin{aligned}
& W_{2}(p \cdot t) \Lambda_{1}(t, p)=\Lambda_{1}(t, p) W_{2}(p), \text { and } \\
& W_{2}^{\perp}(p \cdot t) \Lambda_{1}(t, p)=\Lambda_{1}(t, p) W_{2}^{\perp}(p),
\end{aligned}
$$

i.e. the cocycle $\Lambda_{1}(t, p)$ on $\mathbb{R}^{m}$ defines a flow that leaves $i m Q_{2}$ and $i m Q_{2}^{\perp}$ invariant. Now define for $\lambda \in \mathbb{R}$ a cocycle $\Lambda_{2}^{\lambda}(t, p)$ on $S \times \mathbb{R}^{m}$ through

$$
\begin{aligned}
& \Lambda_{2}^{\lambda}(t, p) W_{2}(p)=G_{2}(p \cdot t) \Phi(t, p) G_{2}^{-1}\left(W_{2}(p)\right), \\
& \Lambda_{2}^{\lambda}(t, p) W_{2}^{\perp}(p)=e^{\lambda t} \Lambda_{1}(t, p) W_{2}^{\perp}(p) .
\end{aligned}
$$

Then $\Lambda_{2}^{\lambda}(t, p)$ satisfies for $p \in S, t \in \mathbb{R}$

$$
\begin{gathered}
W_{2}(p \cdot t) \Lambda_{2}^{\lambda}(t, p)=\Lambda_{2}^{\lambda}(t, p) W_{2}(p), \\
W_{2}^{\perp}(p \cdot t) \Lambda_{2}^{\lambda}(t, p)=\Lambda_{2}^{\lambda}(t, p) W_{2}^{\perp}(p), \\
G_{2}(p \cdot t) \Phi(t, p)=\Lambda_{2}^{\lambda}(t, p) G_{2}(p) .
\end{gathered}
$$


Since $G_{2}$ is an isomorphism between the vector bundles $\pi: E \longrightarrow S$ and $\pi^{\prime}$ : $i m Q_{2} \longrightarrow S$, where $\pi^{\prime}$ is given by $W_{2}^{-1}$, it is a cohomology between $\Phi$ and the flow induced by $\Lambda_{2}^{\lambda}$ on $i m Q_{2}$.

Now choose $\lambda$ large enough such that we obtain for $\Lambda_{2}^{\lambda}$ an exponential dichotomy on $G_{2}(E)=i m Q_{2}$ and $i m Q_{2}^{\perp}=\operatorname{ker} Q_{2}$. Then $\left\{\mathbb{P i m} Q_{2}, \mathbb{P} k e r Q_{2}\right\}$ form a Morse decomposition of $\mathbb{P} \Lambda_{2}^{\lambda}$.

By Lemma 2.2 there exists a smooth linear flow $\Psi$ on $S \times \mathbb{R}^{m}$, which is cohomologous to $\Lambda_{2}^{\lambda}$. Define $E^{\prime}$ and $E^{\prime \prime}$ as the images of $i m Q_{2}$, and $k e r Q_{2}$ respectively, under this cohomology. Then (4.4) and (4.6) follow from Proposition 4.1. Formula (4.5) is a consequence of the Morse decomposition property of $\left\{\operatorname{Pim} Q_{2}, \mathbb{P} k e r Q_{2}\right\}$, which is preserved under cohomology. Finally, (4.7) follows from (4.5) and the fact that $\lambda$ separates the Morse spectra over $i m Q_{2}$ and $\operatorname{ker} Q_{2}$.

According to Theorem 4.2 we can analyze the Morse spectrum of any linear flow $\Phi$ on a vector bundle by considering smooth linear flows $\Psi$ on a trivial bundle $S \times \mathbb{R}^{m}$. These flows allow the use of smooth ergodic theory. We start with some concepts and notations.

Let $(S, d)$ be a metric space, and denote its Borel $\sigma$-algebra by $\mathbb{B}$. A probability measure $\mu$ on $(S, \mathbb{B})$ is called invariant for a flow $\Phi: \mathbb{R} \times S \rightarrow S$ if $\Phi_{t} \mu=\mu$ for all $t \in \mathbb{R}$, i.e. if $\left(\Phi_{t} \mu\right)(A):=\mu\left(\Phi_{t}^{-1} A\right)=\mu A$ for all $A \in \mathbb{B}$. The measure $\mu$ is called ergodic if $\mu\left(A \backslash \Phi_{t}^{-1} A \cup \Phi_{t}^{-1} A \backslash A\right)=0$ for all $t \in \mathbb{R}$ implies $\mu A=1$ or $\mu A=0$.

For a linear flow $\Phi$ on a vector bundle $\pi: E \rightarrow S$ we use the notation

$$
\mathcal{P}(\Phi):=\{\mu ; \mu \text { is an invariant probability measure of } \mathbb{P} \Phi \text { on } \mathbb{P} E\}
$$

and

$$
\mathcal{P}^{e}(\Phi):=\{\mu \in \mathcal{P}(\Phi) ; \mu \text { is ergodic }\} .
$$

4.3. Lemma. Let $\Phi$ and $\Psi$ be linear flows on vector bundles with cohomology $F$. Then

$$
\mathcal{P}(\Psi)=\{\mathbb{P} F \mu ; \mu \in \mathcal{P}(\Phi)\} \quad \text { and } \quad \mathcal{P}^{e}(\Psi)=\left\{\mathbb{P} F \mu ; \mu \in \mathcal{P}^{e}(\Phi)\right\} .
$$

Proof. Note that $\mathbb{P} F, \mathbb{P} \Phi_{t}$ and $\mathbb{P} \Psi_{t}$ are homeomorphisms for all $t \in \mathbb{R}$. Now the result follows from the definitions by repeated use of the commutative diagram (2.1).

Next we generalize the Krylov-Bogolyubov construction of invariant measures to sequences of chains. Let $S$ be a compact metric space with a flow $\Phi$. Let $\zeta$ be an $(\varepsilon, T)$-chain of $\Phi$ on $S$ given by $n \in \mathbb{N}, T_{i} \geq T, p_{i} \in S$ with $i=0 \ldots n-1$. Denote by $C(S)$ the space of real valued continuous functions on $S$, and define a continuous linear functional $\mathcal{A}$ on $C(S)$ by

$$
\mathcal{A} f:=\left(\sum_{i=0}^{n-1} T_{i}\right)^{-1} \sum_{i=0}^{n-1} \int_{0}^{T_{i}} f\left(\Phi\left(t, p_{i}\right)\right) d t .
$$

Note that for $i=0, \ldots, n-1$ the map

$$
f \mapsto \frac{1}{T_{i}} \int_{0}^{T_{i}} f\left(\Phi\left(t, p_{i}\right)\right) d t
$$

defines a Radon probability measure $\nu_{i}$ on $S$. The measure $\nu$ corresponding to $\mathcal{A}$ is a convex combination of the $\nu_{i}$, hence also a Radon probability measure. 
Now consider for $\varepsilon^{k} \rightarrow 0, T^{k} \rightarrow \infty$ a sequence of $\left(\varepsilon^{k}, T^{k}\right)$-chains $\zeta^{k}$, given by $n^{k} \in \mathbb{N}, T_{i}^{k} \geq T^{k}$, and $p_{i}^{k} \in S$ for $i=0 \ldots n^{k}-1, k \in \mathbb{N}$. Define $\mathcal{A}^{k}$ for $\zeta^{k}$ as in (4.8), with corresponding measure $\nu^{k}$. As $k \rightarrow \infty$, a subsequence of $\left\{\nu^{k}, k \in \mathbb{N}\right\}$, denoted again by $\left\{\nu^{k}\right\}$, converges weakly to a Radon probability measure $\mu$ on $S$, i.e. we have for all $f \in C(S)$

$$
\lim _{k \rightarrow \infty}\left(\sum_{i=0}^{n^{k}-1} T_{i}^{k}\right)^{-1} \sum_{i=0}^{n^{k}-1} \int_{0}^{T_{i}^{k}} f\left(\Phi\left(t, p_{i}^{k}\right)\right) d t=\int_{S} f d \mu .
$$

Then, using standard arguments (see e.g. [NS], Theorem VI. 9.05, or [Ma], Section I.8), one shows that the measure $\mu$ is invariant under the flow, i.e. in particular

$$
\int_{S} f(p) d \mu=\int_{S} f\left(\Phi_{t}(p)\right) d \mu \quad \text { for all } \quad t \in \mathbb{R} .
$$

We will use this construction to express the boundary points of the Morse spectrum via ergodic measures.

4.4. Lemma. Let $\Psi$ be a smooth linear flow on the trivial bundle $S \times \mathbb{R}^{d}$, and let $\mathcal{T} \subset S \times \mathbb{P}^{d-1}$ be a compact invariant set for $\mathbb{P} \Psi$. Then there exist ergodic $\mathbb{P} \Psi$-invariant probability measures $\mu^{*}$ and $\mu$ on $S \times \mathbb{P}^{d-1}$ with

$$
\inf \Sigma_{M o}(\mathcal{T}, \Psi)=\int_{\mathcal{T}} q d \mu^{*}, \sup \Sigma_{M o}(\mathcal{T}, \Psi)=\int_{\mathcal{T}} q d \mu,
$$

where $q: S \times \mathbb{P}^{d-1} \rightarrow \mathbb{R}$ is the continuous function defined in (2.3).

Proof. Consider for $\varepsilon^{k} \rightarrow 0, T^{k} \rightarrow \infty$ a sequence of $\left(\varepsilon^{k}, T^{k}\right)$-chains $\zeta^{k}$ in a chain recurrent component $\mathcal{M}$ of $\mathbb{P} \Psi \mid \mathcal{T}$ with

$$
\lambda\left(\zeta^{k}\right) \rightarrow \sup \Sigma_{M o}(\mathcal{T}, \Psi) .
$$

The $\zeta^{k}$ are given by $n^{k} \in \mathbb{N}, T_{i}^{k} \geq T^{k}$, and $\left(p_{i}^{k}, \mathbb{P} v_{i}^{k}\right) \in \mathcal{T} \subset S \times \mathbb{P}^{d-1}$ for $i=$ $0 \ldots n^{k}-1, k \in \mathbb{N}$. Then, by Lemma 2.1

$$
\begin{aligned}
\lambda\left(\zeta^{k}\right) & =\left(\sum_{i=0}^{n^{k}-1} T_{i}^{k}\right)^{-1} \sum_{i=0}^{n^{k}-1}\left(\log \left|\Psi\left(T_{i}^{k}, p_{i}^{k}, v_{i}^{k}\right)\right|-\log \left|v_{i}^{k}\right|\right) \\
& =\left(\sum_{i=0}^{n^{k}-1} T_{i}^{k}\right)^{-1} \sum_{i=0}^{n^{k}-1} \int_{0}^{T_{i}^{k}} q\left(\mathbb{P} \Psi\left(t, p_{i}^{k}, \mathbb{P} v_{i}^{k}\right)\right) d t .
\end{aligned}
$$

By the Krylov-Bogolyubov construction for chains there exists a $\mathbb{P} \Psi$-invariant measure $\mu$ on $\mathcal{M}$ as in (4.9). In particular, since $q$ is continuous, we have

$$
\lambda\left(\zeta^{k}\right) \rightarrow \int_{\mathcal{M}} q d \mu
$$

hence $\mu$ satisfies the second equality in (4.11).

Now assume that all ergodic, $\mathbb{P} \Psi$-invariant measures on $\mathcal{T}$ violate the second equality in (4.11). For $\nu$-almost all $(p, \mathbb{P} v)$ in the support of an ergodic invariant 
measure $\nu$ we have

$$
\lambda(p, v)=\lim _{t \rightarrow \infty} \frac{1}{t} \int_{0}^{t} q(\mathbb{P} \Psi(\tau, p, \mathbb{P} v)) d \tau,
$$

and hence, by our assumption,

$$
\int_{\mathcal{T}} q d \nu<\sup \Sigma_{M o}(\mathcal{T}, \Psi) .
$$

But the invariant measure $\mu$ in (4.13) is in the closed, convex hull of the ergodic invariant measures on $\mathcal{T}$, which contradicts (4.12), (4.13). (Compare e.g. [Ma] Sections I.6 and II.6 for the facts on invariant measures used above.) The existence of $\mu^{*}$ satisfying the first equality in (4.11) is proved similarly.

4.5. Remark. Recall that the set of invariant probability measures for a flow on a compact metric space is convex. Hence Lemma 4.4 shows that every element in the Morse spectrum $\Sigma_{M o}(\Phi)$, and in particular by Theorem 3.7 every Lyapunov exponent can be written as $\int_{S \times \mathbb{P}^{d-1}} q d \mu$ for some $\mathbb{P} \Phi$-invariant probability measure $\mu$.

Combining Lemma 4.4 with Theorem 4.2 we obtain the following characterization of the boundary points of the Morse spectrum for arbitrary linear flows.

4.6. Theorem. Let $\Phi$ be a linear flow on a vector bundle $\pi: E \rightarrow S$, and let $\mathcal{T} \subset \mathbb{P} E$ be a compact, $\mathbb{P} \Phi$-invariant set. Then there exist ergodic, $\mathbb{P} \Phi$-invariant probability measures $\mu^{*}(\mathcal{T})$ and $\mu(\mathcal{T})$ with support in $\mathcal{T}$ such that

$$
\begin{aligned}
& \kappa^{*}(\mathcal{T}):=\inf \Sigma_{M o}(\mathcal{T}, \Phi)=\lim _{t \rightarrow \pm \infty} \frac{1}{t} \log \left|\Phi\left(t, e^{*}\right)\right| \\
& \text { for } \mu^{*}(\mathcal{T}) \text {-almost all } \mathbb{P} e^{*} \in \mathbb{P} E, \\
& \kappa(\mathcal{T}):=\sup \Sigma_{M o}(\mathcal{T}, \Phi)=\lim _{t \rightarrow \pm \infty} \frac{1}{t} \log |\Phi(t, e)| \\
& \text { for } \mu(\mathcal{T}) \text {-almost all } \mathbb{P} e \in \mathbb{P} E .
\end{aligned}
$$

Proof. By Theorem 4.2, there exists a smooth linear flow $\Psi$ on a trivial bundle $S \times \mathbb{R}^{m}$, such that $\Phi$ is cohomologous via $F: E \rightarrow E^{\prime}$ to a subflow $\Psi \mid E^{\prime}$, and (4.4)-(4.7) hold. Since $\mathcal{T}^{\prime}:=\mathbb{P} F(\mathcal{T})$ is compact and $\mathbb{P} \Psi$-invariant, one finds by Lemma 4.4 ergodic invariant measures $\mu_{1}^{*}$ and $\mu_{1}$ with support in $\mathcal{T}^{\prime}$ such that

$$
\kappa^{*}\left(\mathcal{T}^{\prime}\right)=\int_{\mathcal{T}^{\prime}} q d \mu_{1}^{*}, \kappa\left(\mathcal{T}^{\prime}\right)=\int_{\mathcal{T}^{\prime}} q d \mu_{1}
$$

Hence (cf. e.g. [Ma], Theorem II. 6.1) it follows that for $\mu_{1}^{*}$-almost all $\mathbb{P} e^{*} \in \mathbb{P} E^{\prime}$

$$
\kappa^{*}\left(\mathcal{T}^{\prime}\right)=\lim _{t \rightarrow \pm \infty} \frac{1}{t} \int_{0}^{t} q\left(\mathbb{P} \Psi\left(\tau, \mathbb{P} e^{*}\right)\right) d \tau=\lim _{t \rightarrow \pm \infty} \frac{1}{t} \log \left|\Psi\left(t, e^{*}\right)\right|,
$$

and for $\mu_{1}$-almost all $\mathbb{P} e \in \mathbb{P} E^{\prime}$

$$
\kappa\left(\mathcal{T}^{\prime}\right)=\lim _{t \rightarrow \pm \infty} \frac{1}{t} \int_{0}^{t} q(\mathbb{P} \Psi(\tau, \mathbb{P} e)) d \tau=\lim _{t \rightarrow \pm \infty} \frac{1}{t} \log |\Psi(t, e)| .
$$


Lemma 4.3 shows that under $\mathbb{P} F$ the ergodic, invariant measures $\mu_{1}^{*}$ and $\mu_{1}$ correspond to ergodic, invariant measures $\mu^{*}$ and $\mu$ of $\mathbb{P} \Phi$. Furthermore, the proof of Proposition 4.1(ii) yields

$$
\kappa^{*}(\mathcal{T})=\kappa^{*}\left(\mathcal{T}^{\prime}\right) \text { and } \kappa(\mathcal{T})=\kappa\left(\mathcal{T}^{\prime}\right) .
$$

Using again the properties of the cohomology $F$ as in the proof of Proposition 4.1 (ii), we obtain (4.14) and (4.15).

4.7. Remark. A result, analogous to Theorem 4.6, holds also for the boundary points of the dynamical spectrum, cf. [JPS], Theorem 2.3. The proof above follows essentially the same lines.

Next we use a result due to Bronstein and Chernii $[\mathrm{BC}]$ showing that the decomposition (3.2) coincides with the finest decomposition into exponentially separated invariant subbundles, see also Bronstein [Br1], Theorems 7.22 and 7.23. Here 'exponential separation' means that - comparing solutions starting in the same fiber - the exponential growth rate for a solution in the first bundles is (uniformly) smaller than for any solution in the second bundle. This notion is made precise in the following definition.

4.8. Definition. For a linear flow $\Phi$ on a vector bundle $\pi: E \rightarrow S$ a pair of complementary invariant subbundles $\left(\mathcal{V}^{+}, \mathcal{V}^{-}\right)$of $E$ is called exponentially separated, if there are $c>0$ and $\mu>0$ with

$$
\left|\Phi_{t} v^{+}\right| \leq c \exp (-\mu t)\left|\Phi_{t} v^{-}\right|
$$

for all $t \geq 0$ and $v^{+} \in \mathcal{V}^{+}, v^{-} \in \mathcal{V}^{-}$with $\pi\left(v^{+}\right)=\pi\left(v^{-}\right)$and $\left|v^{+}\right|=\left|v^{-}\right|$.

4.9. Theorem. Let $\Phi$ be a linear flow on a vector bundle $\pi: E \rightarrow S$ with chain recurrent flow on the base space $S$. Then the decomposition (3.2) into invariant subbundles corresponding to the finest Morse decomposition of the induced flow on the projective bundle has the following property:

If $\left(\mathcal{V}^{+}, \mathcal{V}^{-}\right)$are exponentially separated subbundles, then there is $1 \leq j \leq l$ such that

$$
\mathcal{V}^{+}=\mathcal{V}_{1} \oplus \cdots \oplus \mathcal{V}_{j} \text { and } \mathcal{V}^{-}=\mathcal{V}_{j+1} \oplus \cdots \oplus \mathcal{V}_{l}
$$

Conversely, if $\mathcal{V}^{+}$and $\mathcal{V}^{-}$are defined by these equalities, then $\left(\mathcal{V}^{+}, \mathcal{V}^{-}\right)$are exponentially separated.

Proof. By Lemma 3 in [BC] (see also Theorem 6.18 in [Br2]), a decomposition into exponentially separated subbundles $\left(\mathcal{V}^{+}, \mathcal{V}^{-}\right)$is equivalent to the fact that $\left(\mathbb{P} \mathcal{V}^{-}, \mathbb{P} \mathcal{V}^{+}\right)$is an attractor-repeller pair. Hence the claim follows from Theorem 3.1 .

Combining this with our previous results we obtain the following corollary.

4.10. Corollary. Let $\Phi$ be a linear flow on a vector bundle $\pi: E \rightarrow S$, and let $\mathcal{M}$ be a chain recurrent component of the projected flow $\mathbb{P} \Phi$. Then

$$
\Sigma_{M o}(\mathcal{M}, \Phi)=\Sigma_{M o}^{p}(\mathcal{M}, \Phi)
$$

Furthermore $\Sigma_{M o}(\Phi)$ consists of closed intervals of (periodic) chain exponents and

$$
\Sigma_{L y}(\Phi) \subset \Sigma_{M o}(\Phi)=\bigcup_{\mathcal{M}}\left[\kappa^{*}(\mathcal{M}), \kappa(\mathcal{M})\right],
$$


whose boundary points are Lyapunov exponents. If the flow on the base space is chain recurrent (or over a chain recurrent component in the base space), then

$$
\Sigma_{M o}(\Phi)=\bigcup_{i=1}^{l} \Sigma_{M o}\left(\mathcal{M}_{i}, \Phi\right)=\bigcup_{i=1}^{l}\left[\kappa^{*}\left(\mathcal{M}_{i}\right), \kappa\left(\mathcal{M}_{i}\right)\right]
$$

where $\mathcal{M}_{i}$ are the chain recurrent components of the flow $\mathbb{P} \Phi$ and $\kappa^{*}\left(\mathcal{M}_{i}\right)<\kappa^{*}\left(\mathcal{M}_{j}\right)$ and $\kappa\left(\mathcal{M}_{i}\right)<\kappa\left(\mathcal{M}_{j}\right)$ for $i<j$.

Proof. Let $\mathcal{M}$ be a chain recurrent component of the projected flow $\mathbb{P} \Phi$. Then, by Theorem 3.6, $\Sigma_{M o}^{p}(\mathcal{M}, \Phi)=\left[\kappa_{p}^{*}(\mathcal{M}), \kappa_{p}(\mathcal{M})\right]$, and $\lambda(e) \in \Sigma_{M o}^{p}(\mathcal{M}, \Phi)$ for all $e \in E$ with $\omega(\mathbb{P e}) \subset \mathcal{M}$ by Theorem 3.7. On the other hand, Theorem 4.6 shows that $\kappa^{*}(\mathcal{M})=\lambda\left(e^{*}\right)$ and $\kappa(\mathcal{M})=\lambda(e)$ for some $e^{*}, e \in E$ with $\omega\left(\mathbb{P} e^{*}\right) \subset \mathcal{M}, \omega(\mathbb{P} e) \subset \mathcal{M}$. Now the obvious inequalities $\kappa^{*}(\mathcal{M}) \leq \kappa_{p}^{*}(\mathcal{M})$ and $\kappa_{p}(\mathcal{M}) \leq \kappa(\mathcal{M})$ imply $(4.16)$. Except for the order of the spectral intervals, the other assertions follow from Theorems 3.1, 3.6 and 4.6.

Now let $i \in\{1, \ldots, l\}$. By Theorem 4.9, the vector bundles $\mathcal{V}_{i}^{+}=\bigoplus_{j=1}^{i} \mathbb{P}^{-1}\left(\mathcal{M}_{j}\right)$ and $\mathcal{V}_{i}^{-}=\bigoplus_{j=i+1}^{l} \mathbb{P}^{-1}\left(\mathcal{M}_{j}\right)$ are exponentially separated. Hence there are numbers $c_{i}>0, \mu_{i}>0$ such that for $e^{+} \in \mathcal{V}_{i}^{+}, e^{-} \in \mathcal{V}_{i}^{-}$with $\pi e^{+}=\pi e^{-}$and $\left|e^{+}\right|=\left|e^{-}\right|=1$ it follows that

$$
\left|\Phi_{t}\left(e^{+}\right)\right| \leq c_{i} \exp \left(-\mu_{i} t\right)\left|\Phi_{t}\left(e^{-}\right)\right|, \quad t \geq 0 .
$$

For $\varepsilon>0, T>0$ consider an $(\varepsilon, T)$-chain $\zeta \subset \mathcal{M}_{i+1}$ given by

$$
n \in \mathbb{N}, T_{0}, \ldots, T_{n-1} \geq T, \mathbb{P} e_{0}, \ldots, \mathbb{P} e_{n} \in \mathcal{M}_{i+1} .
$$

Then the growth rate $\lambda(\zeta)$ is

$$
\lambda(\zeta)=\left(\sum_{k=0}^{n-1} T_{i}\right)^{-1}\left(\sum_{k=0}^{n-1} \log \left|\Phi\left(T_{i}, e_{k}\right)\right|-\log \left|e_{k}\right|\right),
$$

with $e_{k} \in \mathbb{P}^{-1}\left(\mathbb{P} e_{k}\right)$. Clearly, one finds an $(\varepsilon, T)$-chain $\zeta^{\prime} \subset \mathcal{M}_{i}$ given by

$$
n \in \mathbb{N}, T_{0}, \ldots, T_{n-1}, \mathbb{P} e_{0}^{\prime}, \ldots, \mathbb{P} e_{n}^{\prime} \in \mathcal{M}_{i}
$$

with $\pi e_{k}=\pi e_{k}^{\prime}$ for all $k$. Then by exponential separation, the growth rates satisfy

$$
\lambda\left(\zeta^{\prime}\right) \leq \lambda(\zeta)-\mu_{i}
$$

Now approximate $\inf \Sigma_{M o}\left(\mathcal{M}_{i+1}\right)=\inf \Sigma_{M o}\left(\mathcal{V}_{i}^{-}\right)$by $(\varepsilon, T)$-chains $\zeta$ in $\mathcal{M}_{i+1}$. Since there are $(\varepsilon, T)$-chains $\zeta^{\prime}$ in $\mathcal{M}_{i}$ starting in the same fibers, the estimate above shows that

$$
\inf \Sigma_{M o}\left(\mathcal{M}_{i}\right) \leq \inf \Sigma_{M o}\left(\mathcal{M}_{i+1}\right)-\mu_{i}
$$

The assertion for the suprema follows by time reversal (or using analogous arguments).

The rest of this section is devoted to integral representations of Lyapunov exponents that are useful in the study of control flows, see [CK1], [CK2]. 
Consider a linear flow $\Phi$ on a vector bundle $\pi: E \rightarrow S$ and let $\lambda \in \Sigma_{L y}(\Phi)$ be given by

$$
\lambda(e)=\lambda(\mathbb{P} e)=\lim _{k \rightarrow \infty} \frac{1}{t_{k}} \log |\Phi(t, e)| .
$$

Then, by the Krylov-Bogolyubov construction, there exists a $\mathbb{P} \Phi$-invariant measure $\mu$ on $\omega(\mathbb{P e})$, such that for a subsequence, which we denote again by $\left\{t_{k}\right\}$

$$
\lim _{k \rightarrow \infty} \frac{1}{t_{k}} \int_{0}^{t_{k}} f(\mathbb{P} \Phi(\tau, \mathbb{P} e)) d \tau=\int f d \mu
$$

for all $f \in C(\mathbb{P} E)$. In particular, if $\Psi$ is a smooth linear flow on $S \times \mathbb{R}^{m}$, one can take for $f$ the function $q$ from (2.3), and hence

$$
\lambda(e)=\int_{S \times \mathbb{P}^{d-1}} q d \mu .
$$

For an arbitrary linear flow $\Phi$ we can use the cohomology $F$ between $\Phi$ and the subflow $\Psi \mid E^{\prime}$ according to Theorem 4.2 to obtain a continuous function $r: \mathbb{P} E \rightarrow$ $\mathbb{R}, r=q \circ \mathbb{P} F$ and a $\mathbb{P} \Phi$-invariant measure $\nu=(\mathbb{P} F)^{-1} \mu$, which yields with Proposition 4.1 (iii) for every Lyapunov exponent of $\Phi$ an expression of the form

$$
\lambda(e)=\int_{\mathbb{P} E} r d \nu, \quad e \in E .
$$

Often one would like to have a more direct representation of the measure and the integrand. This is possible for flows that satisfy a certain 'absolute continuity' condition, compare e.g. the set up for control flows in [CK1]. The result shows in particular, that every Lyapunov exponent can be obtained as an integral over regular Lyapunov exponents.

4.11. Theorem. Let $\Phi$ be a linear flow on a vector bundle $\pi: E \rightarrow S$, and assume that there exists a bounded (Lebesgue-)measurable function $Q: \mathbb{P E} \rightarrow \mathbb{R}$ such that for all $e \in E, t \in \mathbb{R}$

$$
\log |\Phi(t, e)|-\log |e|=\int_{0}^{t} Q(\mathbb{P} \Phi(\tau, \mathbb{P} e)) d \tau .
$$

Then for each $e \in E$ there exists a $\mathbb{P} \Phi$-invariant probability measure $\mu=\mu(e)$ with $\operatorname{supp} \mu \subset \omega(\mathbb{P} E)$ such that for $\mu$-almost all $\mathbb{P} a \in \mathbb{P} E$ and all $a \in \mathbb{P}^{-1}(\mathbb{P} a)$

$$
\lambda(a)=\lim _{t \rightarrow \pm \infty} \frac{1}{t} \log |\Phi(t, a)|=\lim _{t \rightarrow \pm \infty} \frac{1}{t} \int_{0}^{t} Q(\mathbb{P} \Phi(\tau, \mathbb{P} a)) d \tau,
$$

(in particular, all limits exist) and

$$
\lambda(e)=\int_{\omega(\mathbb{P} e)} Q d \mu=\int_{\omega(\mathbb{P} e)} \lambda d \mu .
$$


Proof. For every invariant measure $\mu$ on $\mathbb{P} E$, the function $Q$ is $\mu$-integrable. Furthermore, for all Lyapunov exponents $\lambda(e)$, given by

$$
\lambda(e)=\lim _{k \rightarrow \infty} \frac{1}{t_{k}} \log \left|\Phi\left(t_{k}, e\right)\right|
$$

it follows from (4.20) that

$$
\lambda(e)=\lim _{k \rightarrow \infty} \frac{1}{t_{k}} \int_{0}^{t_{k}} Q(\mathbb{P} \Phi(\tau, \mathbb{P} e)) d \tau .
$$

By Theorem 4.2 there is a cohomology $F$ between $\Phi$ and a subflow $\Psi \mid E^{\prime}$ of a smooth linear flow $\Psi$ on $S \times \mathbb{R}^{m}$. Thus by (4.18) we obtain

$$
\lambda(e)=\lambda(F e)=\lim _{k \rightarrow \infty} \frac{1}{t_{k}} \int_{0}^{t_{k}} q(\mathbb{P} \Psi(\tau, \mathbb{P} F e)) d \tau=\int_{\mathbb{P} E^{\prime}} q d \nu,
$$

where $\nu=\nu(\mathbb{P} F e)$ is a $\mathbb{P} \Psi$-invariant measure on $\mathbb{P} E^{\prime}$ with supp $\nu \subset \omega(\mathbb{P} F e)$. By Birkhoff's Ergodic Theorem (see e.g. [NS], Section VI. 5 and 6, or [Ma], Theorem II.1.1) we have that for $\nu$-almost all $\mathbb{P} b \in \mathbb{P} E^{\prime}$

$$
\tilde{q}(\mathbb{P} b):=\lim _{t \rightarrow \infty} \frac{1}{t} \int_{0}^{t} q(\mathbb{P} \Psi(\tau, \mathbb{P} b)) d \tau
$$

exists. The function $\tilde{q}$ is $\nu$-integrable and

$$
\int q d \nu=\int \tilde{q} d \nu=\int \lambda(\mathbb{P} b) d \nu
$$

Let $\mu:=(\mathbb{P} F)^{-1} \nu$ be the corresponding $\mathbb{P} \Phi$-invariant measure on $\mathbb{P} E$ (see Lemma $4.3)$, then we obtain using (4.24)

$$
\lambda(e)=\int \lambda(\mathbb{P} F(\mathbb{P} a)) \mu(d \mathbb{P} a) .
$$

Now $F$ is a cohomology, hence by Proposition 4.1(iii)

$$
\lambda(\mathbb{P} F(\mathbb{P} a))=\lambda(\mathbb{P} a)=\lim _{t \rightarrow \infty} \frac{1}{t}|\Phi(t, a)|
$$

for $\mu$-almost all $\mathbb{P} a \in \mathbb{P} E$. This yields for the measure $\mu$

$$
\lambda(e)=\int \lambda(\mathbb{P} a) d \mu
$$

where (4.25) holds for $\mu$-almost all $\mathbb{P} a \in \mathbb{P} E$. Again by Birkhoff's Ergodic Theorem we have that for $\mu$-almost all $\mathbb{P} a \in \mathbb{P} E$

$$
\tilde{Q}(\mathbb{P} a):=\lim _{t \rightarrow \infty} \frac{1}{t} \int_{0}^{t} Q(\mathbb{P} \Phi(\tau, \mathbb{P} a)) d \tau
$$

exists, and

$$
\int \tilde{Q} d \mu=\int Q d \mu
$$

Now (4.25), (4.27) and (4.23) imply (4.21), and (4.22) follows from (4.26) and (4.28), since $\tilde{Q}(\mathbb{P} a)=\lambda(\mathbb{P} a)$ holds $\mu$-almost everywhere. 
4.12. Remark. Specializing the set up of Theorem 4.9 to linearized control flows, we obtain the results of [CK1], Theorem 4.5 (i) and Corollary 4.6 (ii). The proofs of these statements, given in [CK1] are too simplistic.

\section{Relations of the Morse Spectrum to other Spectral Concepts}

In this section we discuss the relation of the Morse spectrum to other spectral concepts, namely the dichotomy spectrum introduced by Sacker and Sell [SS2], a topological concept introduced by Salamon and Zehnder [SZ] (which we call-for lack of a better expression, the topological spectrum), and the Oseledeč spectrum [Os]. A number of interesting connections between the dichotomy spectrum, the Oseledeč spectrum, and the Lyapunov spectrum have been derived in [JPS]. We also remark that exponential separation (see Definition 4.8) is equivalent to the existence of a cocycle such that the linear flow multiplied by this cocycle has an exponential dichotomy, see Palmer [Pa], Bronstein [Br1], and Theorem 6.30 in [Br2].

Let $\Phi$ be a linear flow on a vector bundle $\pi: E \rightarrow S$, and denote

$$
\Phi_{t}^{\lambda}:=\exp (-\lambda t) \Phi_{t}
$$

The topological spectrum of $\Phi$ is defined as (cf. [SZ])

$$
\Sigma_{\text {top }}(\Phi):=\left\{\lambda \in \mathbb{R} \text {; the zero section } Z \text { is not an isolated invariant set of } \Phi_{t}^{\lambda}\right\} .
$$

Thus $\lambda \in \Sigma_{\text {top }}$ if there exists $e \in E \backslash Z$ such that $\exp (-\lambda t) \Phi(t, e)$ is bounded on $\mathbb{R}$.

5.1. Theorem. For a linear flow $\Phi$ on a vector bundle $\pi: E \rightarrow S$ it holds that

$$
\Sigma_{M o}(\Phi) \subset \Sigma_{t o p}(\Phi) .
$$

If the induced flow $\pi \Phi$ on the base space $S$ is chain transitive, then equality holds in (5.2).

Proof. Assume for now that the flow $\pi \Phi$ on $S$ is chain transitive. Then (see Theorem 3.1) there exists a finest Morse decomposition of $\mathbb{P} \Phi$, inducing a Whitney decomposition

$$
E=\mathcal{V}_{1} \oplus \cdots \oplus \mathcal{V}_{\ell}
$$

into $\Phi$-invariant subbundles. By Corollary 4.10 we have

$$
\Sigma_{M o}(\Phi)=\bigcup_{i=1}^{\ell}\left[\kappa^{*}\left(\mathbb{P} \mathcal{V}_{i}\right), \kappa\left(\mathbb{P} \mathcal{V}_{i}\right)\right]
$$

Now suppose that $\lambda \notin \Sigma_{t o p}(\Phi)$, i.e. the zero section $\mathrm{Z}$ is an isolated invariant set for the flow $\Phi_{t}^{\lambda}$. Then, by [SZ], Theorem 2.7 the unstable set

$$
E^{\lambda, u}:=\left\{e \in E ; \phi \neq \omega^{*}(e) \subset Z\right\}
$$

and the stable set

$$
E^{\lambda, s}:=\{e \in E ; \phi \neq \omega(e) \subset Z\}
$$

project down to an attractor-repeller pair $\mathbb{P} E^{\lambda, u}, \mathbb{P} E^{\lambda, s}$ in $\mathbb{P} E$. (Here the limit sets are formed with respect to the flow $\Phi_{t}^{\lambda}$.) Since (5.3) corresponds to a finest Morse decomposition of $\mathbb{P} \Phi=\mathbb{P} \Phi^{\lambda}$, we know that for some $k \in\{1 \ldots \ell\}$

$$
E^{\lambda, u}=\mathcal{V}_{1} \oplus \cdots \oplus \mathcal{V}_{k}, E^{\lambda, s}=\mathcal{V}_{k+1} \oplus \cdots \oplus \mathcal{V}_{\ell},
$$


with an appropriate renumbering of the $\mathcal{V}_{i}$. Hence, by Lemma 3.3 and (5.4)

$$
\Sigma_{M o}(\Phi)=\Sigma_{M o}\left(\Phi \mid E^{\lambda, u}\right) \cup \Sigma_{M o}\left(\Phi \mid E^{\lambda, s}\right) .
$$

Now, since the stable and the unstable set induce a subbundle decomposition of $E$, we can use [SZ], Theorem 2.13 to obtain the existence of an $\varepsilon>0$ such that $\sup \Sigma_{L y}\left(\Phi^{\lambda} \mid E^{\lambda, s}\right)<-\varepsilon$, i.e. $\sup \Sigma_{L y}\left(\Phi \mid E^{\lambda, s}\right)<\lambda-\varepsilon$. Hence, by Theorem 4.6, $\lambda$ cannot be in $\Sigma_{M o}\left(\Phi \mid E^{\lambda, s}\right)$.

Similarly, one sees by time reversal (compare Remark 3.10) that $\lambda$ cannot be in $\Sigma_{M o}\left(\Phi \mid E^{\lambda, u}\right)$, and thus $\Sigma_{M o}(\Phi) \subset \Sigma_{t o p}(\Phi)$.

Conversely, if $\lambda \notin \Sigma_{M o}(\Phi)$, we obtain from (5.3) and (5.4) a decomposition of $E$ into invariant subbundles $\mathcal{V}^{\lambda, u}$ and $\mathcal{V}^{\lambda, s}$ with

$$
\inf \Sigma_{M o}\left(\Phi \mid \mathcal{V}^{\lambda, u}\right)>\lambda+\varepsilon \text { and } \sup \Sigma_{M o}\left(\Phi \mid \mathcal{V}^{\lambda, s}\right)<\lambda-\varepsilon
$$

for some $\varepsilon>0$. Obviously, $\mathbb{P} \mathcal{V}^{\lambda, u}$ is an attractor of $\mathbb{P} \Phi$ in $\mathbb{P} E$, and $\mathbb{P} \mathcal{V}^{\lambda, s}$ its complementary repeller. And for the limit sets of the flow $\Phi^{\lambda}$ it holds that

$$
\begin{aligned}
\phi \neq \omega^{*}(e) \subset Z & \text { for all } e \in \mathcal{V}^{\lambda, u}, \text { and } \\
\phi \neq \omega(e) \subset Z & \text { for all } e \in \mathcal{V}^{\lambda, s} .
\end{aligned}
$$

Hence, using again [SZ], Theorem 2.7 we obtain that the zero section $Z$ is an isolated invariant set of $\Phi^{\lambda}$, i.e. $\lambda \notin \Sigma_{t o p}(\Phi)$.

Now we drop the assumption that the flow $\pi \Phi$ on the base space $S$ is chain transitive. Then, according to Definition 3.2, for each $\lambda \in \Sigma_{M o}(\Phi)$ there exists a chain recurrent component $M \subset S$ of $\pi \Phi$ such that $\lambda \in \Sigma_{M o}\left(\Phi \mid \pi^{-1} M\right)$. By the first part of the proof this implies that $\lambda \in \Sigma_{t o p}\left(\Phi \mid \pi^{-1} M\right)$, i.e. $Z$ is not an isolated invariant set of $\Phi^{\lambda} \mid \pi^{-1} M$. Thus $Z$ is not an isolated invariant set of $\Phi^{\lambda}$, and hence $\lambda \in \Sigma_{\text {top }}(\Phi)$.

In general, the inclusion (5.2) will be strict, as the following example shows (compare [SZ], p. 626).

5.2. Example. Let $\pi: E \rightarrow S$ be given by $E=[0,1] \times \mathbb{R}, S=[0,1]$. Consider the flow induced by

$$
\dot{x}=x(1-x), \dot{z}=\left(\frac{1}{2}-x\right) z \quad \text { with } x \in[0,1], z \in \mathbb{R} .
$$

The chain recurrent set of the projected flow on $[0,1] \times\{1\}$ consists of the two points $\left(x_{0}, \mathbb{P} z_{0}\right)=(0,1)$ and $\left(x_{1}, \mathbb{P} z_{1}\right)=(1,1)$. We obtain by direct calculation

$$
\left\{\frac{1}{2},-\frac{1}{2}\right\}=\Sigma_{M o} \varsubsetneqq \Sigma_{t o p}=\left[0, \frac{1}{2}\right] \cup\left[-\frac{1}{2}, 0\right] \text {. }
$$

The difference, of course, comes from the fact that the Morse spectrum is only concerned with the exponential growth behavior over the chain recurrent components of the base flow, and not with the behavior of the flow between these components. This example also shows that the Lyapunov spectrum may be strictly contained in the topological spectrum.

The following results on the relation between the topological spectrum and the dichotomy spectrum are well known (cf. the references below). For the reader's convenience, we provide the required definitions and sketch some of the arguments.

An exponential dichotomy of a linear flow $\Phi$ on a vector bundle $\pi: E \rightarrow S$ is given by a continuous, fiber preserving map $P: E \rightarrow E$ (such that the induced 
maps $P_{p}: E_{p} \rightarrow E_{p}$ are nontrivial projections for each $p \in S$ ) and by constants $K \geq 1, \alpha>0$ with

$$
\begin{aligned}
\left|\Phi_{t} P \Phi_{s}^{-1}\right| & \leq K \exp (-\alpha(t-s)) & & \text { for } s \leq t, \\
\left|\Phi_{t}(I d-P) \Phi_{s}^{-1}\right| & \leq K \exp (-\alpha(s-t)) & & \text { for } s \geq t .
\end{aligned}
$$

The dichotomy spectrum of the linear flow $\Phi$ is defined by

$$
\Sigma_{\text {dic }}(\Phi)=\left\{\lambda \in \mathbb{R} ; \Phi^{\lambda} \text { has no exponential dichotomy }\right\} .
$$

5.3. Theorem. Let $\Phi$ be a linear flow on a vector bundle $\pi: E \rightarrow S$. Then

$$
\Sigma_{\text {top }}(\Phi) \subset \Sigma_{\text {dic }}(\Phi)
$$

If the induced flow $\pi \Phi$ on the base space $S$ is chain transitive, then equality holds in (5.7).

Proof. Suppose that $\lambda \notin \Sigma_{d i c}(\Phi)$, i.e. there exists an exponential dichotomy for $\Phi^{\lambda}$ which yields a decomposition

$$
E=E^{s} \oplus E^{u}
$$

into invariant subbundles $E^{s}:=P E$ and $E^{u}=(I-P) E$ with

$$
\begin{array}{cc}
\left|\Phi^{\lambda}(t, e)\right| \leq K \exp (-\alpha t) & \text { for } e \in E^{s}, t \geq 0, \text { and } \\
\left|\Phi^{\lambda}(t, e)\right| \leq K \exp (\alpha t) & \text { for } e \in E^{u}, t \leq 0 .
\end{array}
$$

We have to show that the zero section $Z$ is isolated for $\Phi^{\lambda}$. Assume to the contrary that there is $e \in E \backslash Z$ and $\gamma>0$ such that

$$
\left|\Phi^{\lambda}(t, e)\right| \leq \gamma \quad \text { for all } t \in \mathbb{R} .
$$

Pick $\varepsilon>0$ and choose $\tau \geq 0$ with $K \exp (-\alpha \tau) \leq \varepsilon$. If $v \in E^{s}$ satisfies

$$
\left|\Phi^{\lambda}(t, v)\right| \leq \gamma \quad \text { for } t \leq 0,
$$

then

$$
|v|=\left|\Phi^{\lambda}\left(\tau, \Phi^{\lambda}(-\tau, v)\right)\right| \leq K \exp (-\alpha \tau) \gamma \leq \varepsilon \gamma .
$$

Since $\varepsilon$ was arbitrary, it follows that $|v|=0$, i.e. $v \in Z$. Similarly, if $w \in E^{u}$ and

$$
\left|\Phi^{\lambda}(t, w)\right| \leq \gamma \text { for } t \geq 0,
$$

then $w \in Z$.

For an arbitrary $e \in E$ satisfying (5.8) we write $e=v+w, v \in E^{s}, w \in E^{u}$. By linearity we obtain

$$
\left|\Phi^{\lambda}(t, w)\right| \leq\left|\Phi^{\lambda}(t, e)\right|+\left|\Phi^{\lambda}(t, v)\right| .
$$

Since $v \in E^{s},\left|\Phi^{\lambda}(t, v)\right|$ is bounded for $t \geq 0$, hence it follows from (5.8) that $w \in E^{u}$ satisfies (5.10) for some $\gamma>0$, and therefore $w \in Z$. Similarly, $v \in E^{s}$ satisfies (5.9) for some $\gamma>0$, and hence $v \in Z$. This yields $e \in Z$, which implies that $Z$ is isolated for $\Phi^{\lambda}$.

For a chain recurrent base space $S$, equality in (5.7) is the main result on the dichotomy spectrum, see e.g. [S1], Theorem IV. 9 or [SS2].

The proof above follows the ideas of [SS1], Lemma 11(3).

5.4. Remark. It is well known that the inclusion (5.7) may be strict, see e.g. [Ro] p. 435 , also for further references. 
So far we have shown that for a linear flow $\Phi$ on a vector bundle $\pi: E \rightarrow S$ the following chain of inequalities holds

$$
\Sigma_{L y}(\Phi) \subset \Sigma_{M o}(\Phi) \subset \Sigma_{t o p}(\Phi) \subset \Sigma_{d y n}(\Phi) .
$$

In general, the last two inclusions can be strict. A similar result is not known for the Lyapunov and the Morse spectrum. Denote the boundary points of the Morse spectrum by

$$
\partial \Sigma_{M o}(\Phi)=\left\{\kappa^{*}(\mathcal{M}), \kappa(\mathcal{M}) ; \mathcal{M} \text { is a chain recurrent component of } \mathbb{P} \Phi\right\} .
$$

Then we have by Theorem 4.6

$$
\partial \Sigma_{M o}(\Phi) \subset \Sigma_{L y}(\Phi) \subset \Sigma_{M o}(\Phi) .
$$

If we restrict the flow $\Phi$ to a chain recurrent component $M \subset S$ of $\pi \Phi$ (i.e. in particular if $\pi \Phi$ is chain recurrent), then we obtain

$$
\begin{aligned}
\partial \Sigma_{M o}\left(\Phi \mid \pi^{-1} M\right) & \subset \Sigma_{L y}\left(\Phi \mid \pi^{-1} M\right) \subset \Sigma_{M o}\left(\Phi \mid \pi^{-1} M\right) \\
& =\Sigma_{\text {top }}\left(\Phi \mid \pi^{-1} M\right)=\Sigma_{d i c}\left(\Phi \mid \pi^{-1} M\right) .
\end{aligned}
$$

Furthermore, it follows from [SS1], Theorem 4 and [JPS], Theorem 2.3 that

$$
\partial \Sigma_{\text {dic }}\left(\Phi \mid \pi^{-1} M\right) \subset \Sigma_{L y}\left(\Phi \mid \pi^{-1} M\right),
$$

where $\Sigma_{\text {dic }}\left(\Phi \mid \pi^{-1} M\right)$ consists of finitely many disjoint compact intervals in $\mathbb{R}$, and $\partial \Sigma_{d i c}\left(\Phi \mid \pi^{-1} M\right)$ are simply the boundary points of these intervals. Recall from Theorem 3.6 that $\Sigma_{M o}\left(\Phi \mid \pi^{-1} M\right)$ consists of finitely many compact intervals in $\mathbb{R}$ (these are exactly the (periodic) Morse exponents over $M$ ), but these intervals need not be disjoint, as the following example shows.

5.5. Example. Let $E=\mathcal{U} \times \mathbb{R}^{2}$, where $\mathcal{U}:=\{u: \mathbb{R} \rightarrow U$, measurable $\}$ and $U=[-1,1] \times\left[-\frac{1}{4}, \frac{1}{4}\right] \times\left[-\frac{1}{4}, \frac{1}{4}\right] \subset \mathbb{R}^{3}$. Equipped with the weak ${ }^{*}$ topology of $L^{\infty}\left(\mathbb{R}, \mathbb{R}^{3}\right)=L^{1}\left(\mathbb{R}, \mathbb{R}^{3}\right)^{*}, \mathcal{U}$ is a compact metric space. Define a linear flow $\Phi$ on $\mathcal{U} \times \mathbb{R}^{2}$ by $\Phi_{t}(u, x)=\left(\theta_{t} u, \varphi(t, x, u)\right)$ where $\theta_{t}: \mathcal{U} \rightarrow \mathcal{U}, \theta_{t} u(\cdot)=u(t+\cdot)$ and $\varphi(t, x, u)$ is the solution of the differential equation

$$
\dot{x}=\left(\begin{array}{cc}
1 & \frac{3}{4} \\
\frac{3}{4} & 1
\end{array}\right) x+u_{1}(t)\left(\begin{array}{cc}
1 & 0 \\
0 & 1
\end{array}\right) x+u_{2}(t)\left(\begin{array}{ll}
0 & 1 \\
0 & 0
\end{array}\right) x+u_{3}(t)\left(\begin{array}{ll}
0 & 0 \\
1 & 0
\end{array}\right) x
$$

with $\varphi(0, x, u)=x$. The base flow $\theta=\pi \Phi$ on $\mathcal{U}$ is chain recurrent (see [CK3], Lemma 4.5). There are two chain recurrent components of $\mathbb{P} \Phi$ on $\mathcal{U} \times \mathbb{P}^{1}$, namely

$$
\begin{aligned}
& \mathcal{M}_{1}=\mathcal{U} \times \mathbb{P}\left\{\left(\begin{array}{l}
x_{1} \\
x_{2}
\end{array}\right) \in \mathbb{R}^{2} ; x_{2}=\alpha x_{1}, \alpha \in\left[-\sqrt{2},-\frac{1}{\sqrt{2}}\right]\right\}, \\
& \mathcal{M}_{2}=\mathcal{U} \times \mathbb{P}\left\{\left(\begin{array}{l}
x_{1} \\
x_{2}
\end{array}\right) \in \mathbb{R}^{2} ; x_{2}=\alpha x_{1}, \alpha \in\left[\frac{1}{\sqrt{2}}, \sqrt{2}\right]\right\} .
\end{aligned}
$$

The Morse spectrum of $\Phi$ is

$$
\Sigma_{M o}(\Phi)=\Sigma_{M o}\left(\mathcal{M}_{1}, \Phi\right) \cup \Sigma_{M o}\left(\mathcal{M}_{2}, \Phi\right)=\left[-1, \frac{3}{2}\right] \cup\left[\frac{1}{2}, 3\right]=\Sigma_{L y}(\Phi),
$$

compare [CK6], Example 3.2.

This example shows that in

$$
\partial \Sigma_{d i c}\left(\Phi \mid \pi^{-1} M\right) \subset \partial \Sigma_{M o}\left(\Phi \mid \pi^{-1} M\right) \subset \Sigma_{L y}\left(\Phi \mid \pi^{-1} M\right)
$$


also the first inclusion can be strict. The intervals of the dynamical spectrum over $M$ are simply the disjoint intervals of $\Sigma_{M o}\left(\Phi \mid \pi^{-1} M\right)$.

Next we turn to the Oseledec spectrum. Let $\mu$ be an invariant probability measure of the base flow $\pi \Phi$ on $S$. Then there exists $\Gamma \subset S$ with $\mu \Gamma=1$ such that for all $p \in \Gamma$ there are numbers $\lambda_{i}(p), i=1 \ldots r(p) \leq d=\operatorname{dim} E_{p}$ and a (measurable) decomposition $E_{p}=L_{p}^{1} \oplus \cdots \oplus L_{p}^{r(p)}$ such that $\lim _{t \rightarrow \pm \infty} \frac{1}{t}|\Phi(t, e)|=\lambda_{i}(p)$ iff $e \in L_{p}^{i}$. If $\mu$ is ergodic, then $r(p)$ and $\lambda_{i}(p)$ are constant $\mu$-almost everywhere. The Oseledec spectrum of $(\Phi, \mu)$ is defined as (see [Os] or [Ma], Sections IV. 10 and 11).

$$
\Sigma_{O s}(\Phi, \mu)=\left\{\lambda \in \mathbb{R} ; \lambda=\lambda_{i}(p) \text { for some } p \in \Gamma, i=1 \ldots r(p)\right\} .
$$

Obviously, we have

$$
\bigcup_{\mu} \Sigma_{O s}(\Phi, \mu) \subset \Sigma_{L y}(\Phi) \text { where the union is taken over all } \pi \Phi \text { invariant measures. }
$$

5.6. Corollary. Let $\Phi$ be a linear flow on a vector bundle $\pi: E \rightarrow S$. Then

$$
\partial \Sigma_{M o}(\Phi) \subset \bigcup_{\mu} \Sigma_{O s}(\Phi, \mu), \mu \pi \Phi \text {-invariant and ergodic. }
$$

Proof. This is a simple corollary of Theorem 4.6: Let $\mathcal{M}$ be a chain recurrent component of $\mathbb{P} \Phi$. Then there exists an ergodic, $\mathbb{P} \Phi$-invariant measure $\mu^{*}(\mathcal{M})$ such that $\lim _{t \rightarrow \pm \infty} \frac{1}{t} \log \left|\Phi\left(t, e^{*}\right)\right|=\kappa^{*}(\mathcal{M})$ for $\mu^{*}(\mathcal{M})$-almost all $\mathbb{P} e^{*} \in \mathbb{P} E$. Hence the marginal $\mu^{*}:=\mathbb{P} \pi\left(\mu^{*}(\mathcal{M})\right)$ is $\pi \Phi$-invariant and ergodic, and $\kappa^{*}(\mathcal{M}) \in \Sigma_{O s}\left(\Phi, \mu^{*}\right)$. A similar argument holds for $\kappa(\mathcal{M})$, compare also [JPS], p. 23.

Putting things together we obtain

$$
\begin{aligned}
& \partial \Sigma_{d i c}\left(\Phi \mid \pi^{-1} M\right) \subset \partial \Sigma_{M o}\left(\Phi \mid \pi^{-1} M\right) \subset \bigcup_{\mu \text { erg. }} \Sigma_{O s}\left(\Phi \mid \pi^{-1} M, \mu\right) \\
& \subset \bigcup_{\mu \text { stat }} \Sigma_{O s}\left(\Phi \mid \pi^{-1} M, \mu\right) \subset \Sigma_{L y}\left(\Phi \mid \pi^{-1} M\right) \subset \Sigma_{M o}\left(\Phi \mid \pi^{-1} M\right) .
\end{aligned}
$$

5.7. Remark. Under certain conditions it can be shown that the Morse spectrum of $\Phi$ agrees with the closure of the Floquet spectrum, i.e. the closure of the periodic Lyapunov spectrum, see e.g. Section 6 . below or [CK6], Section 5. In these cases the last three inclusions of (5.17) are equalities.

Finally, we analyze the subbundle decompositions of $\pi: E \rightarrow S$ that are associated with the various spectral concepts. We assume for now that the base flow $\pi \Phi$ is chain recurrent, i.e. we work on a component of $\pi \Phi$.

The Morse spectrum is constructed in such a way that the Morse sets $\mathcal{M}_{1}, \ldots$, $\mathcal{M}_{\ell}, \ell \leq d=\operatorname{dim} E_{p}$, of $\mathbb{P} \Phi$ correspond to the spectral intervals $\left[\kappa^{*}\left(\mathcal{M}_{i}\right), \kappa\left(\mathcal{M}_{i}\right)\right]$, $i=1 \ldots \ell$, and hence $\Sigma_{M o}(\Phi)$ is associated with a continuous Whitney decomposition $E=\mathcal{V}_{1} \oplus \cdots \oplus \mathcal{V}_{\ell}$, such that $\left\{\mathbb{P} \mathcal{V}_{1} \ldots \mathbb{P} \mathcal{V}_{\ell}\right\}$ form the finest Morse decomposition of $\mathbb{P} \Phi$, see Theorem 3.1.

The decomposition of $E$ into stable and unstable subbundles that is associated with the topological spectrum (cf. [SZ], Theorem 2.7, and the proof of Theorem 5.1 above) projects down to attractor-repeller pairs in $\mathbb{P} E$. Hence this decomposition is coarser than the one induced by the finest Morse decomposition. The same holds true for the invariant subbundles of an exponential dichotomy, compare e.g. [SS2]. 
In fact, it is easy to see that the following holds: Let $\mathcal{M}_{j}, i \in I$, be Morse sets of $\mathbb{P} \Phi$ such that $R:=\bigcup_{j \in I} \Sigma_{M o}\left(\mathcal{M}_{j}, \Phi\right)$ is a (maximal) interval of the set $\bigcup_{i=1}^{\ell} \Sigma_{M o}\left(\mathcal{M}_{i}, \Phi\right)$. Then $R$ is an interval of the topological and the dichotomy spectrum, and $\bigoplus_{j \in I} \mathcal{V}_{j}$ is the corresponding spectral bundle. Example 5.5 shows that the bundle decomposition of the Morse spectrum can be strictly finer that the one induced by the topological (or the dichotomy) spectrum. It is this property of the Morse spectrum that allows the characterization of stabilizability of control systems, see e.g. [CK7].

If the base flow $\pi \Phi$ on $S$ is not chain recurrent, then the topological spectrum is still associated with attractor-repeller pairs on $\mathbb{P} E$, (cf. [SZ], Theorem 2.7), and the dichotomy spectrum corresponds to subbundle decomposition via the projectors of exponential dichotomies, but the decompositions corresponding to the Morse spectrum are not defined globally.

The Oseledeč spectrum is defined for $\pi \Phi$-invariant measures $\mu$ on $S$. Hence, for a given $\mu$, the associated (measurable) bundle decomposition can be finer than the one induced by the Morse spectrum. One of the main results of [JPS] shows that for all ergodic $\mu$ the Oseledeč spaces are contained in the subbundles induced by the dichotomy spectrum. As observed by Latushkin in [La] this and Bronstein's result Theorem 6.30 in [Br2] on the relation between exponential separation and exponential dichotomy imply that for an ergodic invariant measure $\nu$ on the base space the Oseledeč bundles are contained in the Morse spectral bundles. Of course, all statements on the Oseledeč spaces hold with $\mu$-probability one.

The following example (the linear oscillator) shows that the number of measurable subbundles obtained from Oseledeč' theorem may be strictly greater than the number of Morse sets and hence of the corresponding subbundles.

5.8. Example. Consider the family of matrices

$$
\left(\begin{array}{rr}
0 & 1 \\
-1 & 0
\end{array}\right)+u(t)\left(\begin{array}{ll}
0 & 0 \\
1 & 0
\end{array}\right) \quad \text { with } u(t) \in U=[-3,3] .
$$

The projected system has one chain control set $E=\mathbb{P}^{1}$, hence one interval of the Morse spectrum $\Sigma_{M o}=\Sigma_{M o}(E)$. Consider the ergodic invariant measure $\delta_{u_{0}}$ on $\mathcal{U}$, the Dirac measure at the point $u_{0} \equiv 2$. The Oseledeč spectrum consists of two points $\Sigma_{O s}\left(\delta_{u_{0}}\right)=\{-1,1\}$ with corresponding Oseledeč spaces

$$
V_{1}=\left\{\left(\begin{array}{r}
x \\
-x
\end{array}\right) ; x \in \mathbb{R}\right\} \text { and } V_{2}=\left\{\left(\begin{array}{l}
x \\
x
\end{array}\right) ; x \in \mathbb{R}\right\} \text {. }
$$

\section{The Lyapunov Spectrum of Smooth Vectorfields with Hyperbolic Projective Flow}

The Morse spectrum of a flow allows the characterization of the Lyapunov spectrum if all $(\varepsilon, T)$-chains for sufficiently small $\varepsilon$ and sufficiently large $T$ can be closed. In fact, Corollary 4.10. implies that closing of the appropriate periodic chains is sufficient to yield that the Floquet spectrum and the Morse spectrum agree. One tool to find trajectories close to $(\varepsilon, T)$-chains is Bowen's Shadowing Lemma (see e.g. $[\mathrm{FS}]$, or $[\mathrm{Ak}]$ ). In this section we will use a strengthened version of the Shadowing Lemma to analyze the Lyapunov spectrum of smooth vector fields with hyperbolic projective flow. For control flows associated with nonlinear control systems (see 
e.g. [CK5]), local accessibility provides another tool to find shadowing orbits close to $(\varepsilon, T)$-chains, see [CK6] for the bilinear case.

The setup for this section is as follows: Let $M$ be a compact, Riemannian $C^{\infty}$ manifold of dimension $d<\infty$, and let $X$ be a $C^{\infty}$-vectorfield on $M$. We denote by $\Phi$ the flow on $M$ generated by $X$, by $T \Phi$ the linearized flow on the tangent bundle $T M$, and by $\mathbb{P} \Phi$ the induced flow on the projective bundle $\mathbb{P} M$. Define the Morse spectrum and the Lyapunov spectrum of $\Phi$ as in Definition 3.2, and in (3.9), respectively. (Note that in Section 3. the linear flow on a vector bundle was denoted by $\Phi$, which corresponds to $T \Phi$ in the current setup.) The Floquet spectrum of $\Phi$ is defined as

$\Sigma_{F \ell}(\Phi):=\{\lambda(\gamma) ;$ there exists a trajectory $T \Phi(t, e)$ in $T M$ such

$$
\text { that } \left.\mathbb{P} \Phi(\cdot, e)=: \gamma \text { is periodic in } \mathbb{P} M \text {, and } \lambda(\gamma):=\lim _{t \rightarrow \infty} \frac{1}{t} \log |T \Phi(t, e)|\right\} .
$$

Recall that a flow $\Psi$ generated by a $C^{\infty}$-vector field on a compact, Riemannian manifold $M$ is called hyperbolic on a compact invariant set $\Lambda \subset M$, if the tangent flow $T \Psi$ on $T M$ leaves invariant a continuous splitting $T_{\Lambda} M=E^{s} \oplus E^{c} \oplus E^{u}$, such that for some $0<\alpha<1$ and some adapted Riemannian metric $|\cdot|$

$$
\begin{array}{cc}
|T \Psi(t, w)|>\alpha^{-t}|w| & \text { for } w \in E^{u} \text { and } t>0, \\
|T \Psi(t, v)|<\alpha^{t}|v| & \text { for } v \in E^{s} \text { and } t>0, \\
E^{c} \text { is the linear span of the vector field. }
\end{array}
$$

The following theorem is the main result of this section.

6.1. Theorem. Let $\Phi$ be the flow generated by the vector field $X$ on $M$. Let $\mathcal{T} \subset \mathbb{P} M$ be a compact $\mathbb{P} \Phi$-invariant set in $\mathbb{P} M$ and assume that $\mathbb{P} \Phi$ is hyperbolic on $\mathcal{T}$. Then

$$
\Sigma_{M o}(\mathcal{T}, \Phi) \subset c \ell \Sigma_{F \ell}(\Phi) \subset c \ell \Sigma_{L y}(\Phi) .
$$

For the proof of Theorem 6.1 we need a version of the (continuous time) Shadowing Lemma. Recall that the continuous time version (in contrast to the case of iterations of diffeomorphisms) involves a reparametrization of time, and we need that this reparametrization is arbitrarily close to the identity. In the following we indicate how the proof given in [FS] has to be modified to yield an appropriate version for our purposes.

First, we recall some notions. For a flow $\Psi$ on a Riemannian manifold $M$ as above we write $\Psi(t, y)=y \cdot t$. The set $y \cdot \mathbb{R}=\{y \cdot t ; t \in \mathbb{R}\}$ for $y \in M$ is called an orbit. A reparametrization of an orbit is an orientation preserving homeomorphism of $\mathbb{R}$, fixing the origin. An $(\varepsilon, T)$-chain given by $n \in \mathbb{N}, T_{0} \ldots T_{n-1} \geq T, x_{0} \ldots x_{n} \in N$ will be written in the form

$$
\begin{aligned}
& x_{0} * t, t \in\left[0, \sum_{i=0}^{n-1} T_{i}\right], \quad \text { where } \\
& x_{0} * t=x_{i} \cdot\left(t-\sum_{j=0}^{i-1} T_{j}\right) \quad \text { for } \sum_{j=0}^{i-1} T_{j} \leq t<\sum_{j=0}^{i} T_{j},
\end{aligned}
$$

analogously for $(\varepsilon, T)$-chains with infinitely many jumps. An orbit $y \cdot \mathbb{R}$ is said to $\delta$-trace an infinite $(\varepsilon, T)$-chain $x_{0} * \mathbb{R}$ if there exists a reparametrization $g$ such that 
$d\left(x_{0} * t, y \cdot g(t)\right)<\delta$ for all $t \in \mathbb{R}$, and analogously on subintervals of $\mathbb{R}$ by restricting $g$.

Our version of the Shadowing Lemma is as follows (cf. [FS], Theorem 4.1.).

6.2. Proposition. Let $\Lambda$ be a hyperbolic compact invariant set of the flow $\Psi$ on $M$. Then for all $\alpha>0$, all $\varepsilon>0$ small enough, all $N>2$ and all $T \geq M$ the following holds: For each periodic $(\varepsilon, T)$-chain $x_{0} *\left[0, \tau_{0}\right]$ in $\Lambda$ there exists a $\tau_{1}$ periodic trajectory $x_{1} \cdot\left[0, \tau_{1}\right]$ and a parametrization $g$ such that $x_{1} \cdot\left[0, \tau_{1}\right] \alpha$-traces $x_{0} *\left[0, \tau_{0}\right]$ via $g$ and furthermore

$$
\begin{aligned}
& |g(t)-t|<\frac{1}{N}(2 t+3) \quad \text { for } t \in\left[0, \tau_{0}\right], \\
& \left|g\left(\tau_{0}\right)-\tau_{1}\right|<\frac{1}{2 N} .
\end{aligned}
$$

The proof of Proposition 6.2 will be established using two lemmas.

6.3. Lemma. Let $\Lambda$ be a hyperbolic compact invariant set of the flow $\Psi$. Then there exists a compact neighborhood $U(\Lambda)$ whose maximal compact invariant subset $\Lambda^{\prime}$ is hyperbolic, and for each $\delta>0$ there is an $\varepsilon>0$ such that each $(\varepsilon, 1)$-chain $x_{0} *[a, b]$ in $\Lambda$ (with $\left.0 \in[a, b]\right)$ is $\delta$-traced by some $y \in \Lambda^{\prime}$. Here for each $N \in \mathbb{N}$ one can choose $y$ and the parametrization $h$ such that for all $t \in[a, b]$

$$
\left(1-\frac{1}{2 N}\right) t+\frac{1}{2 N} a-\frac{1}{N}<h(t)<\left(1+\frac{1}{2 N}\right) t-\frac{1}{2 N} a+\frac{1}{N}
$$

Proof. The proof follows the one of Lemma 3.1. in [FS], where $N=2$ was used. On p. 29, line -9 in [FS] the constant $\gamma$ must be chosen such that $0<\gamma<\frac{1}{N}$. Then one obtains for the parametrization $g(t), t \in[0, r M]$ (cf. [FS], pp. 31-32):

$$
|g(t)-t| \leq(k+1) \gamma \leq \frac{t}{2 N}+\frac{1}{N} \quad \text { for } t \in[k M,(k+1) M], \text { using } M>2 .
$$

Thus

$$
\left(1-\frac{1}{2 N}\right) t-\frac{1}{N}<g(t)<\left(1+\frac{1}{2 N}\right) t+\frac{1}{N} \quad \text { for } t \in[0, r M] .
$$

For a chain $x_{0} *[a, b], \delta$-trace the chain $\left(x_{0} * a\right) *[0, b-a]$ with initial point $x_{0} * a$ by $\{y \cdot g(t), t \in[0, b-a]\}$ for some $y \in \Lambda^{\prime}$. Extend $g$ by $g(t):=t$ for $t<0$. Then the given chain $x_{0} *[a, b]$ can be $\delta$-traced by $\{y \cdot g(-a) \cdot h(t), t \in[a, b]\}$ with $h(t):=g(t-a)-g(-a)$. We find

$$
\begin{aligned}
h(t) & =g(t-a)-g(-a) \\
& <\left(1+\frac{1}{2 N}\right)(t-a)+\frac{1}{N}+a \\
& =\left(1+\frac{1}{2 N}\right) t-\frac{1}{2 N} a+\frac{1}{N} .
\end{aligned}
$$

The estimate from below follows similarly.

6.4. Lemma. For each $\delta>0$ there is an $\varepsilon>0$ such that each infinite $(\varepsilon, 1)$-chain in $\Lambda$ is $\delta$-traced by some $y \in \Lambda^{\prime}$. Here for each $N \in \mathbb{N}$ one can choose $y$ and the parametrization $g$ such that

$$
\begin{aligned}
& |g(t)-t|<\frac{1}{N}(2 t+3) \quad \text { for } t \in \mathbb{R}, \text { and } \\
& g(N)>\frac{1}{2} .
\end{aligned}
$$


Proof. This result follows as in the proof of [FS], Lemma 3.4. using the estimates from Lemma 6.3: The parametrizations $g_{i}$, constructed in [FS] to $\delta_{2}$-trace $x_{i} *[-i, i]$, $i \geq N$ can be chosen to satisfy for $N>2$

$$
g_{i}(N)>\left(1-\frac{1}{2 N}\right) N-\frac{N}{2 N}-\frac{1}{N}=\frac{2 N^{2}-2 N-2}{2 N}>\frac{1}{2}
$$

and this property is inherited by the parametrization $g$, constructed from the $g_{i}$. Furthermore, for $t \in(i, i+1]$ we obtain

$$
\begin{aligned}
\left|g_{i+1}(t)-t\right| & <\frac{1}{2 N} t-\frac{1}{2 N}(-i-1)+\frac{1}{N} \\
& <\frac{1}{2 N} t+\frac{1}{2 N} i+\frac{1}{2 N}+\frac{1}{N} \\
& <\frac{1}{N}(2 t+3),
\end{aligned}
$$

which proves the corresponding statement for the parametrization $g$.

6.5. Proof of Proposition 6.2. Choose $N>2$ and $\tau:=\frac{1}{2 N}$. Then Lemma 3.3 in [FS] guarantees that there exists $\delta>0$ such that, if $x, y \in \Lambda$ are $g, h$ reparametrizations of $x \cdot \mathbb{R}$, and $y \cdot \mathbb{R}$ respectively, with $d(x \cdot g(t), y \cdot h(t))<\delta$ for all $t \in \mathbb{R}$, then $x=y \cdot s$ for some $|s|<\tau$.

Given $\alpha>0$, let $\delta_{1}:=\min \left\{\frac{\delta}{2}, \alpha\right\}$ and let $\varepsilon$ be the corresponding number from Lemma 6.4. Take a periodic $(\varepsilon, T)$-chain $x_{0} *\left[0, \tau_{0}\right]$ in $\Lambda$ with $T \geq N$ and continue it $\tau_{0}$-periodically in both directions to get an infinite $(\varepsilon, T)$-chain through $x_{0}$. Let $\left\{x_{1} \cdot g(t) ; t \in \mathbb{R}\right\} \delta_{1}$-trace the chain $x_{0} * \mathbb{R}$ with parametrization $g$ satisfying

$$
|g(t)-t|<\frac{1}{N}(2 t+3) \text { for } t \in \mathbb{R}, \text { and } g(N)>\frac{1}{2}
$$

according to Lemma 6.4, which shows (6.2). Since $x_{0} * \tau_{0}=x_{0}$, the reparametrization

$$
h(t):=g\left(\tau_{0}-t\right)-g\left(\tau_{0}\right)
$$

has the property that $\left\{\left(x_{1} \cdot g\left(\tau_{0}\right)\right) \cdot h(t) ; t \in \mathbb{R}\right\}$ also $\delta_{1}$-traces $x_{0} * \mathbb{R}$. Hence

$$
d\left(\left(x_{1} \cdot g\left(\tau_{0}\right)\right) \cdot h(t), x_{1} \cdot g(t)\right)<\delta \text { for all } t \in \mathbb{R} .
$$

Now, Lemma 3.3 in [FS] implies as above that there exists $s$ with $|s|<\tau \leq \frac{1}{2}$ and $x_{1}=\left(x_{1} \cdot g\left(\tau_{0}\right)\right) \cdot s$. Since $g\left(\tau_{0}\right) \geq g(N)>\frac{1}{2}$, we obtain $g\left(\tau_{0}\right)+s \neq 0$, and hence $x_{1}$ is a $\tau_{1}$-periodic point with $\tau_{1}:=g\left(\tau_{0}\right)+s,|s|<\tau=\frac{1}{2 N}$. This proves (6.3).

6.6. Proof of Theorem 6.1. It suffices to show that for every $\beta>0$, all $\varepsilon>0$ small enough, all $N \in \mathbb{N}$ large enough, and every periodic $(\varepsilon, T)$-chain $\zeta=x_{0} *\left[0, \tau_{0}\right]$ in $\mathcal{T}$ with $T>N$, there exists a periodic trajectory $x_{1} \cdot \mathbb{R}$ of $\mathbb{P} \Phi$ in $\mathbb{P} M$ with

$$
\left|\lambda(\zeta)-\lambda\left(x_{1}\right)\right|<\beta .
$$


Since the flow $\mathbb{P} \Phi$ is smooth on the Riemannian manifold $\mathbb{P} M$ there exists a continuous function $q: \mathbb{P} M \rightarrow \mathbb{R}$ (see e.g. [Ca], Lemma 3.3), such that

$$
\begin{aligned}
\lambda(\zeta) & =\left(\sum_{i=0}^{n-1} T_{i}\right)^{-1} \sum_{i=0}^{n-1}\left(\log \left|\Phi\left(T_{i}, x_{i}\right)\right|-\log \left|x_{i}\right|\right) \\
& =\left(\sum_{i=0}^{n-1} T_{i}\right)^{-1} \sum_{i=0}^{n-1} \int_{0}^{T_{i}} q\left(\mathbb{P} \Phi\left(t, x_{i}\right)\right) d t \\
& =\frac{1}{\tau_{0}} \int_{0}^{\tau_{0}} q\left(x_{0} * t\right) d t .
\end{aligned}
$$

We apply Proposition 6.2 to the chain $\zeta$ on $\mathbb{P} M$ and obtain for all $\alpha>0$, all $N>2$ a $\tau_{1}$-periodic solution $x_{1} \cdot t, t \in \mathbb{R}$ of $\mathbb{P} \Phi$ with (6.2) and (6.3). Furthermore,

$$
\begin{aligned}
\left|\tau_{0}-\tau_{1}\right| & \leq\left|\tau_{0}-g\left(\tau_{0}\right)\right|+\left|g\left(\tau_{0}\right)-\tau_{1}\right| \\
& <\frac{1}{N}\left(2 \tau_{0}+3\right)+\frac{1}{2 N} \\
& <\frac{3}{N} \tau_{0} .
\end{aligned}
$$

Since $q$ is continuous on the compact space $\mathbb{P} M,|q|$ is bounded, say by $K>0$. Now pick $\beta>0$. Choosing $\alpha>0$ small enough, we obtain by continuity of $q$ and by Proposition 6.2

$$
\left|\int_{0}^{\tau_{0}} q\left(x_{0} * t\right) d t-\int_{0}^{\tau_{0}} q\left(x_{1} \cdot t\right) d t\right|<\frac{1}{3} \tau_{0} \beta,
$$

and therefore

$$
\begin{aligned}
\mid \lambda(\zeta) & -\lambda\left(x_{1}\right)|=| \frac{1}{\tau_{0}} \int_{0}^{\tau_{0}} q\left(x_{0} * t\right) d t-\frac{1}{\tau_{1}} \int_{0}^{\tau_{1}} q\left(x_{1} \cdot t\right) d t \mid \\
& \leq\left|\frac{1}{\tau_{0}} \int_{0}^{\tau_{0}} q\left(x_{0} * t\right) d t-\frac{1}{\tau_{0}} \int_{0}^{\tau_{0}} q\left(x_{1} \cdot t\right) d t\right| \\
& +\left|\frac{1}{\tau_{0}} \int_{0}^{\tau_{0}} q\left(x_{1} \cdot t\right) d t-\frac{1}{\tau_{0}} \int_{0}^{\tau_{1}} q\left(x_{1} \cdot t\right) d t\right| \\
& +\left|\frac{1}{\tau_{0}} \int_{0}^{\tau_{1}} q\left(x_{1} \cdot t\right) d t-\frac{1}{\tau_{1}} \int_{0}^{\tau_{1}} q\left(x_{1} \cdot t\right) d t\right| \\
& \leq \frac{\beta}{3}+\frac{\left|\tau_{1}-\tau_{0}\right|}{\tau_{0}} \cdot K+\frac{\left|\tau_{1}-\tau_{0}\right|}{\tau_{0} \cdot \tau_{1}} \cdot \tau_{1} K .
\end{aligned}
$$

By (6.4) we have $\left|\tau_{1}-\tau_{0}\right| / \tau_{0}<\frac{3}{N}$, and hence for $N$ large enough we obtain

$$
\left|\lambda(\zeta)-\lambda\left(x_{1}\right)\right| \leq \frac{\beta}{3}+\frac{\beta}{3}+\frac{\beta}{3}=\beta
$$

This concludes the proof of Theorem 6.1. 
The following result is an easy consequence of Theorems 6.1, 3.7 and Corollary 4.10 .

6.7. Corollary. Let $\Phi$ be the flow generated by a $C^{\infty}$ vector field on $M$. If all chain recurrent components of $\mathbb{P} \Phi$ on $\mathbb{P} M$ are hyperbolic, then

$$
c \ell \Sigma_{F \ell}(\Phi)=c \ell \Sigma_{L y}(\Phi)=c \ell \Sigma_{M o}(\Phi) .
$$

Note, that if $\Phi$ has a finest Morse decomposition on $M$, then by Theorems 3.1 and 3.6 we have $c \ell \Sigma_{M o}(\Phi)=\Sigma_{M o}(\Phi)$.

6.8. Remark. The hyperbolicity conditions for $\mathbb{P} \Phi$ on $\mathbb{P} M$ and for $\Phi$ on $M$ are not related. This is easily seen by considering linear time invariant differential equations. Here hyperbolicity on $\mathbb{R}^{d}$ means that there is no eigenvalue with vanishing real part, while hyperbolicity in projective space means that the real parts of the eigenvalues are different.

6.9. Remark. We are only aware of one alternative proof for the shadowing lemma in continuous time, due to Coomes, Kocak, and Palmer [CKP]. The statement is given for equations in $\mathbb{R}^{n}$ only, but avoids a reparametrization of time and the proof is more geometrical. It can almost immediately be applied to yield a proof of Theorem 6.1. One only has to observe that - by uniqueness - the shadowing property for arbitrary chains implies that periodic chains are traced by periodic trajectories.

\section{REFERENCES}

[Ak] Akin, Ethan, The General Topology of Dynamical Systems, American Mathematical Society, Graduate Studies in Mathematics 1 (1993). MR 94f:58041

[AN] Arnold, L., D.C. Nguyen, Generic properties of Lyapunov exponents, Random\& Computational Dynamics 2 (1994), 335-345. MR 95m:28018

[Br1] Bronstein, I.U, Transversality implies structural stability, Soviet Math. Dokl. 23 (1981), 251-254, (= Dokl. Akad. Nauk SSSR 257 (1981), 257-268). MR 82h:58038

[Br2] Bronstein, I.U., Nonautonomous Dynamical Systems (1984), Kishinev (in Russian).

[BC] Bronstein, I.U., V.F. Chernii, Linear extensions satisfying Perron's condition. I, Differential Equations 14 (1978), 1234-1243. MR 80c:54046 (Russian original)

[Ca] Carverhill, A., Flows of stochastic dynamical systems: ergodic theory, Stochastics 14 (1985), 273-317. MR 87c:58059

[CK1] Colonius, F., W. Kliemann, Lyapunov exponents of control flows, in Lyapunov Exponents, Arnold, L., H. Crauel, J.-P. Eckmann (eds.), Springer LN Mathematics 1486 (1991), 331-365. MR 93f:93118

[CK2] Colonius, F., W. Kliemann, Remarks on ergodic theory of stochastic flows and control flows, in Diffusion Processes and Related Problems in Analysis, Vol. II, M. Pinsky and V. Wihstutz (eds.), Birkhäuser (1991), 203-240. MR 93i:60110

[CK3] Colonius, F., W. Kliemann, Some aspects of control systems as dynamical systems, J. Dynamics Diff. Equations 5 (1993), 469-494. MR 94g:93063

[CK4] Colonius, F., W. Kliemann, Linear control semigroups acting on projective space, J. Dynamics Diff. Equa. 5 (1993), 495-528. MR 94g:93064

[CK5] Colonius, F., W. Kliemann, Limit behavior and genericity for nonlinear control systems, J. Differential Equations 109 (1994), 8-41. CMP 94:11

[CK6] Colonius, F., W. Kliemann, The Lyapunov spectrum of families of time-varying matrices, Trans. Amer. Math. Soc. 348 (1996), 4389-4408.

[CK7] Colonius, F., W. Kliemann, Asymptotic null-controllability of bilinear systems, Geometry in Nonlinear Control and Differential Inclusions (Warsaw, 1993), Banach Center Publ., 32, Polish Acad. Sci., Warsaw, 1995, 139-148. CMP 96:05

[CKP] Coomes, B.A., H. Kocak, K.J. Palmer, A shadowing theorem for ordinary differential equations, Z. Angew. Math. Phys. 46 (1995), 85-106. MR 96b:58085 
[Cn] Conley, C., Isolated Invariant Sets and the Morse Index, CBMS Regional Conference Series, no. 38, American Mathematical Society, Providence (1978). MR 80c:58009

[Cp] Coppel, W.A., Dichotomies in Stability Theory, Lecture Notes in Mathematics, Vol. 629, Springer-Verlag, 1978. MR 58:1332

[DK] Daleckii, Ju.L., M.G. Krein, Stability of Solutions of Differential Equations in Banach Space, Translations of Math. Monographs, Vol. 43, Amer. Math. Soc., Providence, R.I. (1974). MR 50:5126

[EJ] Ellis, R., R. Johnson, Topological dynamics and linear differential systems, J.Diff. Equations 44 (1982), 21-39. MR 83c:54058

[FS] Franke, J.E., J.F. Selgrade, Hyperbolicity and chain recurrence, J.Diff. Equations 26 (1977), 27-36. MR 57:7685

[HPPS] Hirsch, M., J. Palis, C. Pugh, M. Shub, Neighborhoods of hyperbolic sets, Invent. Math. 9 (1970), 121-134. MR 41:7232

[JPS] Johnson, R.A., K.J. Palmer, G.R. Sell, Ergodic properties of linear dynamical systems, SIAM J. Math. Anal. 18 (1987), 1-33. MR 88a:58112

[Ka] Karoubi, M., K-Theory. An Introduction, Springer-Verlag, 1978. MR 58:7605

[La] Latushkin, Y., Exact Lyapunov exponents and exponentially separated subbundles, Partial Differential Equations, J. Wiener and J. Hale (eds.), Pitman, (1992), 91-95.

[LY] Ledrappier, F., L.-S. Young, Stability of Lyapunov exponents, Ergod. Th. Dynam. Sys. 11 (1991), 469-484. MR 92i:58096

[Ma] Mañé, R., Ergodic Theory and Differentiable Dynamics, Springer-Verlag, 1987. MR 88c: 58040

[MS] Massera, J.L., J.J. Schaeffer, Linear differential equations and functional analysis, Ann. of Math 67 (1958), 517-573. MR 88c:58040

[NS] Nemytskii, V.V., V.V. Stepanov, Qualitative Theory of Dynamical Systems, Princeton University Press, (1960). (Russian edition 1949). MR 22:12258

[Os] Oseledec, V.I., A multiplicative ergodic theorem. Lyapunov characteristic numbers for dynamical systems, Trans. Moscow Math. Soc. 19 (1968), 197-231. MR 39:1629

[Pa] Palmer, K.J., Exponential separation, exponential dichotomy and spectral theory for linear systems of ordinary differential equations, J. Diff. Equations 46 (1982), 324-345. MR 84e:34067

[Ro] Robinson, C., Stability theorems and hyperbolicity in dynamical systems, Rocky Mountain Journal of Mathematics 7 (1977), 425-437. MR 58:13200

[SS1] Sacker, R.J., G.R. Sell, Existence of dichotomies and invariant splittings for linear differential systems 1, J. Diff. Equations 15 (1974), 429-458. MR 49:6209

[SS2] Sacker, R.J., G.R. Sell, A spectral theory for linear differential systems, J. Diff. Equations 37 (1978), 320-358. MR 58:18604

[SZ] Salamon, D., E. Zehnder, Flows on vector bundles and hyperbolic sets, Trans. Amer. Math. Soc. 306 (1988), 623-649. MR 89f:58112

[Sl] Sell, G.R., Lectures on Linear Differential Systems, School of Mathematics, University of Minnesota, Minneapolis, Minnesota, (1975).

[Sg] Selgrade, J., Isolated invariant sets for flows on vector bundles, Trans. Amer. Math. Soc. 203 (1975), 359-390. MR 51:4322

[Si] Sinai, Ya.G. (ed.), Dynamical Systems II, Encyclopedia of Mathematical Sciences, Springer-Verlag, 1989. MR 91i:58079

Institut für Mathematik, Universität Augsburg, 86135 Augsburg, Germany

E-mail address: colonius@uni-augsburg.de

Department of Mathematics, Iowa State University, Ames, Iowa 50011

E-mail address: kliemann@iastate.edu 